\title{
Giant Magnetoelectric Coupling and Magnetic Field-Induced Permanent Switching in a Spin Crossover Mn(III) Complex
}

\author{
Vibe B. Jakobsen ${ }^{\dagger}$, Shalinee Chikara ${ }^{\ddagger}$, Jie-Xiang Yus ${ }^{\S}$ Emiel Dobbelaar ${ }^{\dagger \ltimes}$, Conor T. Kelly ${ }^{\dagger}$, Xiaxin \\ Ding $\neq \S$, Franziska Weickert ${ }^{\ddagger} \%$, Elzbieta Trzop ${ }^{\perp}$, Eric Collet ${ }^{\perp}$, Hai-Ping Cheng§, Grace G. Morgan ${ }^{\dagger *}$, and \\ Vivien S. Zapf** \\ † School of Chemistry, University College Dublin, Belfield, Dublin 4, Dublin, Ireland \\ ‡ National High Magnetic Field Lab, Los Alamos National Laboratory, Los Alamos, NM 87545, USA \\ $\S$ Dept of Physics, University of Florida, Gainesville, FL 32611, USA \\ ${ }^{\perp}$ Univ. Rennes, CNRS, IPR (Institut de Physique de Rennes), UMR 6251, F-35000 Rennes, France
}

\begin{abstract}
We investigate giant magnetoelectric coupling at a $\mathrm{Mn}^{3+}$ spin crossover in $\left[\mathrm{Mn}^{\mathrm{III}} \mathrm{L}\right] \mathrm{BPh} 4\left(\mathrm{~L}=(3,5-\mathrm{diBr}-\mathrm{sal}){ }_{2} 323\right)$ with field-induced permanent switch of the structural, electric and magnetic properties. An applied magnetic field induces a $1^{\text {st }}$ order phase transition from a high spin/low spin (HS-LS) ordered phase to a HS-only phase at $87.5 \mathrm{~K}$ that remains after the field is removed. We observe this unusual effect for DC magnetic fields as low as $8.7 \mathrm{~T}$. The spin-state switching driven by the magnetic field in the bistable molecular material is accompanied by a change in electric polarization amplitude and direction due to a symmetry-breaking phase transition between polar space groups. The magnetoelectric coupling occurs due to a $\gamma \eta^{2}$ coupling between the order parameter $\gamma$ related to the spin-state bistability, and the symmetry-breaking order parameter $\eta$, responsible for the change of symmetry between polar structural phases. We also observe conductivity occurring during the spin crossover, and evaluate the possibility that it results from conducting phase boundaries. We perform ab-initio calculations to understand the origin of the electric polarization change as well as the conductivity during the spin crossover. Thus we demonstrate a giant magnetoelectric effect with a field-induced electric polarization change that is $1 / 10$ of the record for any material.
\end{abstract}

\section{Introduction}

Magnetoelectric (ME) coupling occurs when magnetic field $H$ influences the electric polarization $P$ and dielectric constant, and/or the electric field $E$ influences the magnetization $M$. Applications include electric control of magnetic qubits, as well as magnetic sensors, data storage, and tunable antennas and other frequency devices. ${ }^{1-5} \mathrm{ME}$ coupling is sought after in insulating materials since the large power dissipation from electric currents in conducting materials is eliminated.2, 6-8 To date, the necessary materials to satisfy all needed applications of ME coupling have not been found. Traditionally, it has been common to study ME coupling in inorganic oxides with ordered magnetic spin orientations, such as (anti)ferromagnets. ${ }^{9-10}$ Here we demonstrate ME coupling in a molecule-based spin crossover (SCO) complex exhibiting magnetic-field driven spin state bistability.

In general, SCO occurs in transition-metal complexes with $\mathrm{d}^{4}-\mathrm{d}^{7}$ electrons, such as the $\mathrm{S}=1$ to $\mathrm{S}=2$ transition in JahnTeller active $\mathrm{Mn}^{3+}{ }^{\mathbf{1 1 - 1 8}} \mathrm{SCO}$ is attractive for ME coupling since it can trigger large changes in lattice parameters due to the change in the orbital occupancy of d-shell ions. SCO complexes containing $\mathrm{Mn}^{3+}$ are particularly interesting due to the additional strain from Jahn-Teller effects in the HS state. SCO behavior is common mostly between $\sim 50$ and $400 \mathrm{~K}$ in metal complexes with organic ligands since they can often accommodate the strain. ${ }^{13,16,18-20}$

Most metal-organic complex that exhibit SCO are paramagnets, e.g. there is no magnetic exchange that is significant compared to the energy scales of the SCO. However, spin crossover materials can show cooperative phase transitions since the spin states can couple to each other via strain. ${ }^{16,21-22}$

ME coupling in SCO materials is a relatively new field and has been reported in a few compounds including Mn(taa) $)^{23-25}(H$-induced $\Delta P),\left[\mathrm{Fe}(\mathrm{Htrz})_{2}(\operatorname{trz})\right]\left(\mathrm{BF}_{4}\right)^{26}$ and
$\left[\mathrm{Fe}\left(\mathrm{H}_{2} \mathrm{~B}(\mathrm{pz})_{2}\right)_{2} \text { (bipy) }\right]^{27}$, (E-induced $\left.\Delta M\right)$. Another compound $\left[\mathrm{Fe}(\mathrm{bpp})_{2}\right]^{2+}$ shows ferroelectric and spin crossover behavior though coupling has not yet been demonstrated. ${ }^{28}$ Magnetic field-induced SCO has a longer history and has been studied in a number of materials. Most of these exhibit partial SCOs at very high magnetic fields $>30 \mathrm{~T}$ achieved by pulsed magnets. ${ }^{25,}$ 29-35 Recently, sub-nm SCO devices have been demonstrated with very fast switching speeds and no degradation after $10^{7}$ cycles above room temperature, making these attractive for switching applications. ${ }^{36-37}$ SCOs in hybrid configurations with ferroelectrics or piezoelectrics are also a route to ME coupling, analogous to similar work in oxides. ${ }^{38-40}$

Here we report ME coupling at a cooperative SCO at a relatively low field of $8.7 \mathrm{~T}$ in mononuclear $\mathrm{Mn}^{3+}$ complex $\left[\mathrm{Mn}(3,5-\text { diBr-sal })_{2} 323\right] \mathrm{BPh}_{4} \quad$ (abbreviated $\quad\left[\mathrm{Mn}^{\mathrm{III}} \mathrm{L}\right] \mathrm{BPh}_{4}$ (1) ${ }^{41}$ The SCO is coupled to a structural phase transition between different polar space groups, resulting in a very large ME coupling. This material was previously reported to show an incomplete thermal SCO centered at $86 \mathrm{~K}$ between an $\mathrm{S}=1 / \mathrm{S}=2$ state (HS-LS) and a higher temperature $(>90$ $\mathrm{K})$ pure $\mathrm{S}=2$ (HS) state of $\mathrm{Mn}^{3+}$. The low-temperature phase is a polar $P 1$ phase with a structurally-ordered 1:1 ratio of LS and HS $\mathrm{Mn}^{3+}$ sites, while the higher temperature phase in space group Pc contains two $\mathrm{HS} \mathrm{Mn}^{3+}$ sites (Figure 1a-c). An additional $C c$ HS phase forms above $250 \mathrm{~K}$. We present electric polarization and magnetization experiments on single crystals in DC fields up to $13 \mathrm{~T}$ as well as millisecond pulsed fields up to $65 \mathrm{~T}$ along with ab-initio calculations of the magnitude and directions of the electric polarization vector (Figure 1a-c).

\section{Results and Discussion}

Magnetization and electric polarization 
The magnetic susceptibility of a single crystal of (1), plotted as $\chi_{\mathrm{M}} T$ vs $T$, manifests a SCO as a sharp increase in $\chi_{\mathrm{M}} T$ centered at $88 \mathrm{~K}$ on warming $\left(\mathrm{T}_{1 / 2} \uparrow=88 \mathrm{~K}\right)$ and at $86 \mathrm{~K}$ on cooling $\left(\mathrm{T}_{1 / 2} \downarrow=86 \mathrm{~K}\right)$. Values of $\chi_{\mathrm{M}} T$ are consistent with the HS-LS and HS spin states (Figure 2a). ${ }^{41} M(H)$ was measured at $T=87.5 \mathrm{~K}$ in DC fields while $H$ was swept from $0 \rightarrow$ $13 \rightarrow-13 \rightarrow 13 \rightarrow 0 \mathrm{~T}$. The Brillouin functions in the HS-LS and HS states are shown as dotted lines. The slight deviation from the data is consistent with demagnetization factors in this irregular crystal with sharp corners. This data shows that after an applied magnetic field switches the system to the HS state, the sample remains in this HS state even if the magnetic field is removed as long as temperature remains unchanged (Figure 2b).

$H$-induced SCO behavior has most often been reported above $30 \mathrm{~T}$, achieved with capacitor-driven pulsed magnets. ${ }^{24,29,34,42}$ Thus, the observation of SCO behavior as low as $8.7 \mathrm{~T}$ is unusual. ${ }^{25}$ Moreover, the hysteretic 'trapping' of the HS state is also a significant finding. Such trapping is usually only observed when these materials are exposed to light (light-induced excited spin-state trapping - LIESST) ${ }^{43-}$ ${ }^{45}$ In the case of complex (1) the spin state is switched with a magnetic field in a permanent hysteretic fashion. This permanent switching can occur in two scenarios: (1) the system is initially in the ordered spin (HS-LS) state in zero field at $87.5 \mathrm{~K}$, but that state is a metastable excited state of the free energy at zero field. The magnetic field then assists the transition towards the HS stable state, which remains permanent after the field removed. Alternatively, in scenario (2) the HS-LS ground state initially present at zero field transitions to a new HS ground state in applied $H$. After removal of $H$ the system remains trapped in the now metastable excited HS state. The latter case could be termed magnetic field-induced spin-state trapping (MIESST).

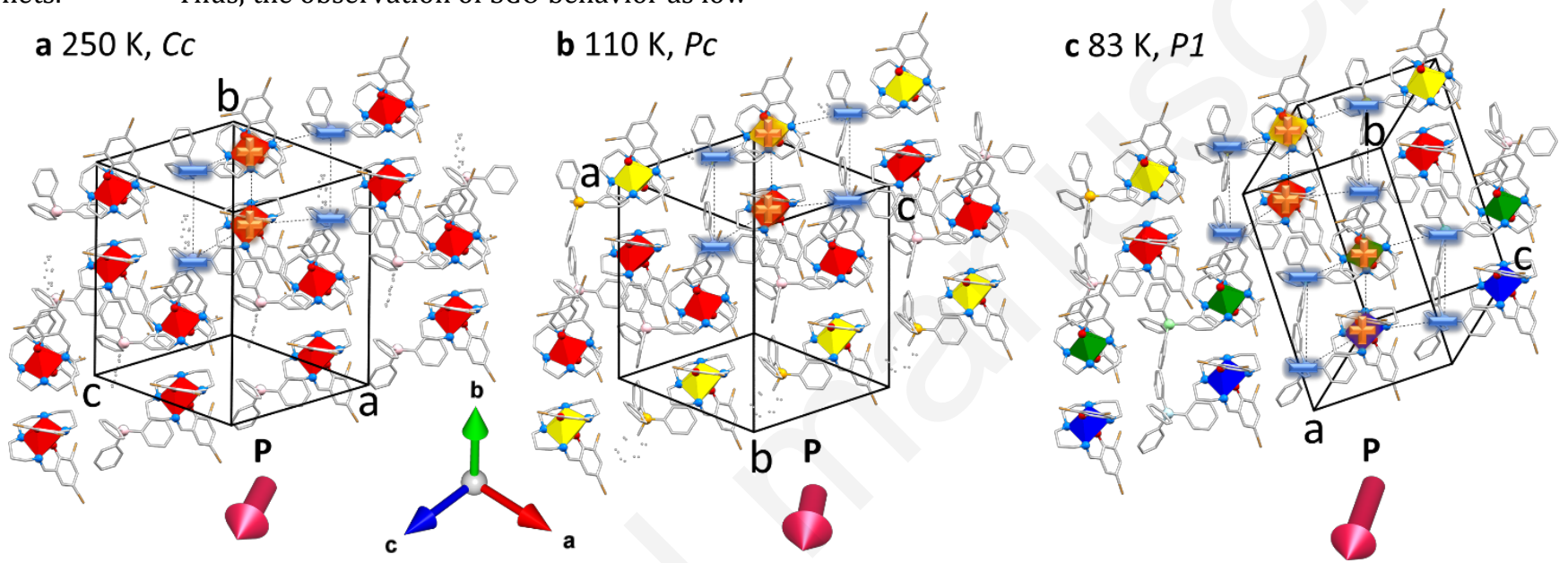

Figure 1. Packing diagrams of [Mn"IIL]BPh 4 (1) with a, HS Cc phase measured at $250 \mathrm{~K}, \mathbf{b}$, HS $P c$ phase measured at $110 \mathrm{~K}, \mathbf{c}, \mathrm{HS}$-LS $P 1$ phase measured at $83 \mathrm{~K} . \mathrm{Mn}^{3+}$ atoms are represented as polyhedra made up of 6 chelating donor atoms in $\mathbf{a}, \mathbf{b}$, c, color-coded according to spin state as red ( $S=2)$, yellow $(S=2)$, blue $(S=1)$ and green $(S=1)$. Structures are shown as stick figures. Donor atoms and the boron atom in the counteranion $\mathrm{BPh}_{4}{ }^{-}$are shown as ball-and-stick figures. Hydrogen atoms are omitted for clarity. The ionic centres are labelled $(+$, orange) and $(-$, blue) respectively. The thin dashed quadrilaterals show the distance between the cationic and anionic part of the molecule. The calculated direction and relative magnitude of bulk electric polarization $P$ in the same reference frame (shown by the inset cartesian coordinate axes) for all three phases is represented by the pink arrows below each subfigure and has a component along the $b$ axis only in the $P 1$ phase. The packing diagrams with $P$ in other orientations can be found in Figures S1-S3 in the Supporting Information. 

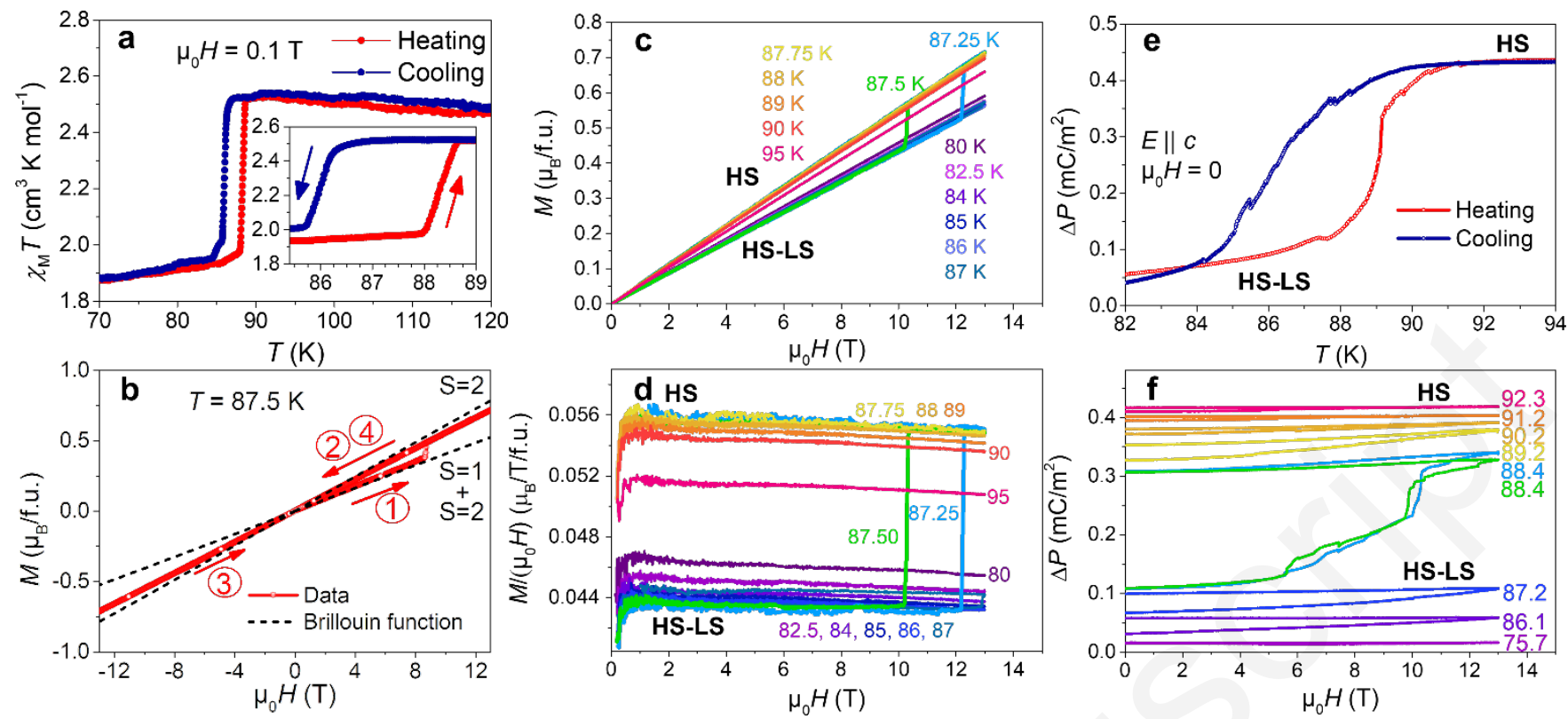

Figure 2. a, $\chi_{\mathrm{M}} T$ vs $T$ of a single crystal for $H / / c=0.1 \mathrm{~T}$ with a zoomed view in the inset. b, $M$ vs $\mu_{0} H$ (red points) showing a hysteretic $H$-induced SCO. Data was taken at $T=87.5 \mathrm{~K}$ with a history of warming from $70 \mathrm{~K}$, measured for $H$ swept from 0 T to $13 \mathrm{~T}(1), 13 \mathrm{~T}$ to $-13 \mathrm{~T}(2),-13 \mathrm{~T}$ to $13 \mathrm{~T} \mathrm{(3)}$ and $13 \mathrm{~T}$ to $0 \mathrm{~T}$ (4) at $0.01 \mathrm{~T} \mathrm{~s}^{-1}$. The Brillouin functions for $\mathrm{HS} \mathrm{S}=2$ and for the HS-LS ordered state with 1:1 ratio of the $\mathrm{S}=1$ and the $\mathrm{S}=2$ states are shown as dotted lines. The data shows the SCO from the HS-LS ordered state to the HS state at $8.7 \mathrm{~T}$ on the first upsweep, and then the system remains in the HS state for the subsequent field sweeps. c, $M\left(\mu_{0} H\right)$ from $0 \mathrm{~T}-13 \mathrm{~T}-0 \mathrm{~T}$ at different temperatures $T$ as indicated. $M\left(\mu_{0} H\right)$ is measured with a history of warming from $70 \mathrm{~K}$ to the desired $T$ as shown. d, The same data plotted as $M / \mu_{0} H$ for clarity. The $H$-induced SCO behavior can be seen at $87.5 \mathrm{~K}$ and $87.25 \mathrm{~K}$ (thicker lines). e, $\Delta P$ vs $T$ at $H=0 . \mathbf{f}, \Delta P$ vs $\mu_{0} H$ under the same experimental conditions of warming from $70 \mathrm{~K}$. At $88.4 \mathrm{~K}, \Delta P$ is shown twice to demonstrate the reproducibility of the switching. The jumps during the transition in $\Delta P$ are not noise - rather they are observed in several different crystals and are likely due to polar domain reorientations. All data was measured with the long axis of the crystal, the $c$ axis in phase $C c$, parallel to the field. Different samples were used in $\mathbf{a}, \mathbf{b}$ and $\mathbf{c}-\mathbf{f}$ resulting in slightly different critical fields.

One example was shown in a Figure by Nakano et al. ${ }^{25}$ but was not investigated further. Such switching should however be a general feature inside the region of bistability of the temperature hysteresis.

We further investigate the $H$-induced switching at different $T$ in Figure 2c-d. The data was taken with a history of warming from $70 \mathrm{~K}$ to the measurement $T$, while data with a cooling history from $110 \mathrm{~K}$ is shown in Figure S6 of the Supporting Information (SI). The data demonstrate that the switching occurs for $T=87.25$ and $87.5 \mathrm{~K}$, in the immediate vicinity of $T_{\mathrm{c}}$.

We have considered and eliminated several potential artefacts that could contribute to the permanent switching: $T$ was kept stable during the measurement; the data is independent of the $H$ sweep rate for $\mathrm{d} H / \mathrm{d} t=2 \mathrm{mTs}^{-1}, 5 \mathrm{mT} \mathrm{s}^{-1}$ and $10 \mathrm{mT} \mathrm{s}^{-1}$ (see Figure S7-S8, Supporting Information); and the possibility of substrate strain contributing to the hysteresis was eliminated by measuring loose crystals, as described in the Supporting Information.

The ME coupling is investigated in Figure 2e-f, showing the electric polarization change $\Delta P$ along the $c$-axis that accompanies the SCO as a function of $T$ and $H$ respectively. Similar to $M, \Delta P$ shows trapping of the HS state after removal of $H$. The steps and breadth of the transition in $P$ were reproduced in several samples - they may result from polar domain reorientations. Indeed, in the $P c$ HS phase, $P$ lies in the ac plane, while in the $P 1$ phase two ferroelastic domains form with an additional component of $P$ along $\pm b$ due to the loss of the glide plane. 

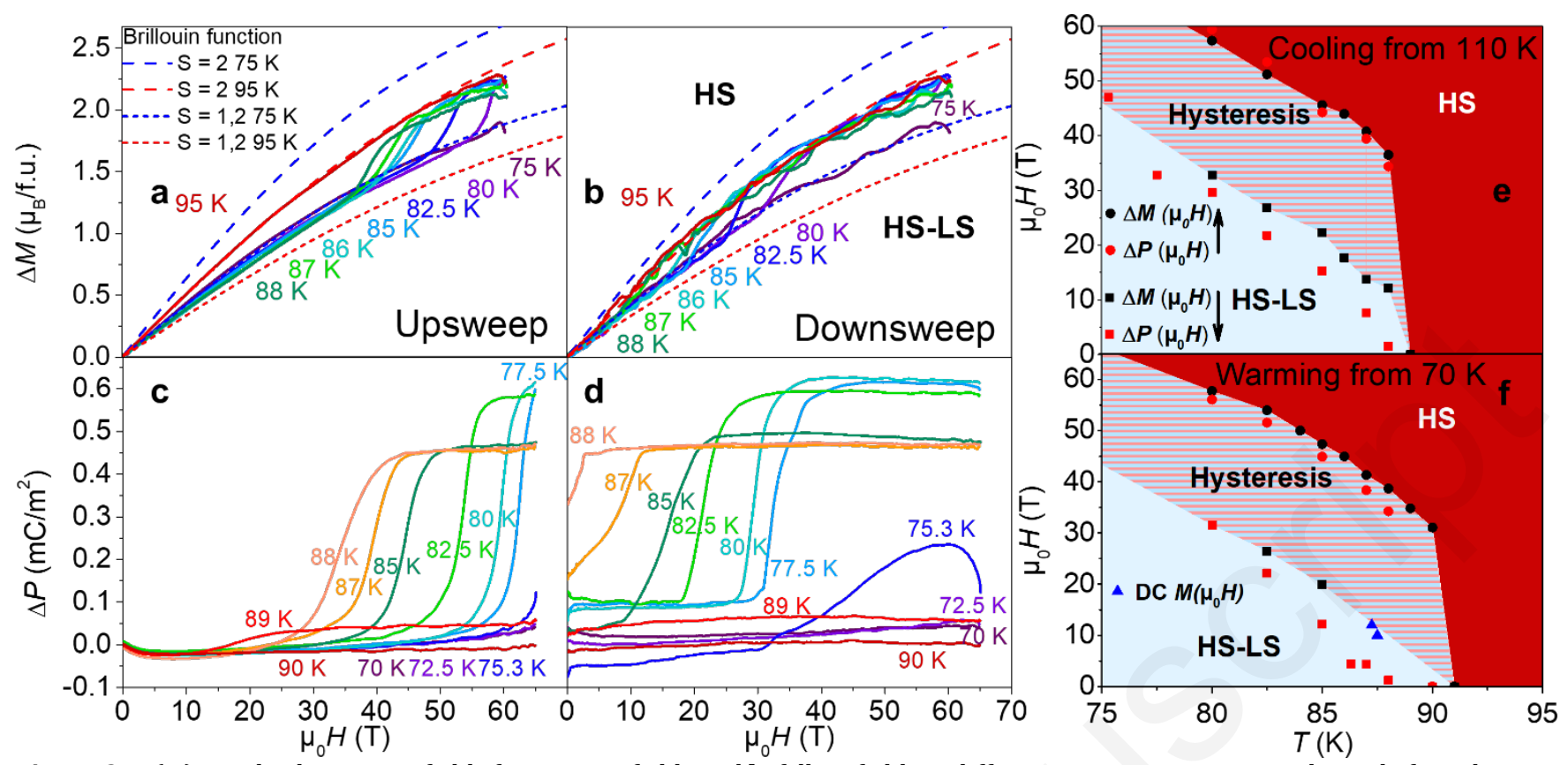

Figure 3. $M(H)$ in pulsed magnetic fields for $\mathbf{a}$, rising fields and $\mathbf{b}$, falling fields at different temperatures $T$ as indicated after a history of cooling from $110 \mathrm{~K}$. Dashed lines are Brillouin functions for the HS states $(\mathrm{S}=2)$ with the upper blue and lower red lines for a given spin state corresponding to the lower and higher $T$, respectively. Similarly, short dashed lines show the Brillouin functions for the HS-LS ordered state. c, $\Delta P(H)$ in a pulsed magnet for $\mathbf{c}$, rising fields and $\mathbf{d}$, falling fields. Data is measured after a history of cooling from $110 \mathrm{~K}$ to the final measurement $T$ between every magnetic field pulse. Corresponding data with warming history is shown in Figure S9-S10. e, Pulsed field H-T phase diagram showing the HS-LS ordered and HS states, and the region of hysteresis in the 1 st order $H$-induced phase transition. Data is shown for a history of cooling from $110 \mathrm{~K}$ and warming from $70 \mathrm{~K}$ for $\mathbf{f}$. Data points in the phase diagram are determined from the midpoint in the $H$ of the transition for pulsed field polarization (red dots) and magnetization curves (black dots) respectively. Arrows in the legend indicate whether $H$ is sweeping up or down. Oscillations in the downsweep data are due to mechanical vibrations.

Notably $\triangle P(H)$ in (1) is giant - of the same order of magnitude as in $\mathrm{Mn}(\mathrm{taa}),{ }^{24}$ significantly larger than that of most molecule-based magnetic materials, ${ }^{46-51}$ and about $1 / 10$ of the record for any material. ${ }^{52}$ Additional electric polarization and dielectric constant data are shown in the Supporting Information along with an extended discussion of how artefacts are ruled out.

Magnetic and electric properties in high fields using capacitor-driven pulsed magnets were also measured in order to explore the phase diagram over a broader $T$ and $H$ range. Figure 3 shows $\Delta M(H)$ and $\Delta P(H)$ up to 65 T. In Figure 3a,b, $\Delta M(H)$ is shown for upsweeps and downsweeps of $H$ together with the Brillouin functions expected in the HS-LS ordered and HS states. Here the high field can induce SCO also outside the thermal region of bistability, down to $75 \mathrm{~K}$ in $\mu_{0} H$ up to 65 T. $\Delta P(H)$ in pulsed fields is shown for up- and downsweeps of the field (Figure 3c,d). $\Delta M(H)$ and $\Delta P(H)$ show transitions consistent with each other.

In agreement with the DC field data, we observe an apparent permanent switching effect in the data with a history of warming. However, in evaluating $H$-induced switching effects, we note that whereas we can be certain of $T$ stability in DC measurements by comparing data taken with different long temperature stabilization times, we cannot guarantee $T$-stability in pulsed fields since there is limited time to thermalize the sample. The SCO can generate heating or cooling due to entropy changes or irreversible heating effects. Thus, we report $H$-induced switching in DC fields but do not confirm it pulsed fields.

\section{Phase Diagram}

The phase transitions in DC and pulsed fields are summarized in the $H-T$ phase diagram in Figure 3e,f. The $H$-induced SCO and its accompanying ME effects can be observed for two Tregimes: (1) within the region of bistability and (2) outside it. Within the bistable region, the slope of the $H-T$ phase boundary is steepest. Since it is a $1^{\text {st }}$ order phase transition, the two phases have similar energies separated by an energy barrier. A change in $H$ or $T$ then alters the free energy landscape until it becomes energetically favorable for the new phase to overcome the barrier, nucleate and propagate.

The second $T$ regime occurs outside the region of bistability, well below $T_{1 / 2}$. Here, the slope of the $H-T$ phase boundary abruptly becomes much shallower. In this $T$ regime, there is a free energy difference between the LS/HS and the HS state that must be closed by the Zeeman effect, which determines the slope of the phase diagram. Once the magnetic field closes the free energy gap the mechanism described in the previous paragraph, takes over. These two mechanisms can be expected to create different slopes in the $H$-T phase diagram. From a mean-field extrapolation we estimate that $H$ needed to induce the SCO at zero $T$ in pulsed fields is $\sim 150 \mathrm{~T}$. Finally we note that in the compound 
Mn(taa) and other SCO materials with $1^{\text {st }}$ order phase transitions, the transitions in pulsed magnets are pushed to higher fields than in DC magnets due to the competition between the speed of the pulse and the speed of domain nucleation and growth. $24,53-60$

One particularity of the present system is that we observe ME coupling in a system with two coupled order parameters: (1.) the high spin fraction $\gamma$ and (2.) the symmetry-breaking structural order parameter $\eta$ that describes the HS-LS order, and which is associated here with a ferroelastic shear as the HS-LS order implies the loss of the $c$ glide plane. In the HS phase, $\gamma=1$ and $\eta=0$, while in the HS-LS phase the symmetry-allowed $\gamma \eta^{2}$ coupling term stabilizes $\gamma$ $=1 / 2$ and $\eta \neq 0.41,61-62$ It is the coupling between the spin state and the structure that causes the SCO to become a $1^{\text {st }}$ order phase transition, as opposed to a gradual crossover. In the present case, $\eta$ allows for $\Delta P$ along $a, b$, and $c$. As $H$ drives switching from $\gamma=1 / 2$ to $\gamma=1, \gamma \eta^{2}$ also tunes a symmetry change between the HS-LS ordered phase and the HS phase and consequently the orientation of $P$.

\section{Calculations of the electric polarization}

The origin of the measured $\Delta P$ along the $c$ axis is a structural phase transition between polar space groups during which molecules tilt and distort, with the Jahn-Teller distortion being a dominant contribution. ${ }^{41}$ Due to symmetry rules, the polarization vector must be in the $a, c$ plane of the HS $C c$ and $P c$ structures. As the $c$ glide plane is lost in the HSLS $P 1$ structure, the lattice vector $b$ is no longer perpendicular to $a$ and $c$, which allows the polarization along $b$ to become non-zero and of opposite sign between the ferroelastic domains.

Table 1. Calculated $\triangle P$ of (1) in the coordinate system of the HS $C c$ phase for all three structural phases. The values are given along the principal axes of the HT phase.

\begin{tabular}{|l|lll|}
\hline Phase & $(\mathrm{HS}-\mathrm{LS})$ & $(\mathrm{HS})$ & $(\mathrm{HS})$ \\
\hline Temperature & $4-83 \mathrm{~K}$ & $83-250 \mathrm{~K}$ & $>250 \mathrm{~K}$ \\
\hline Lattice & Triclinic & Monoclinic & Monoclinic \\
\hline Space Group & $P 1(1)$ & $P c(7)$ & $C c(9)$ \\
\hline$P_{\mathrm{a}}\left(\mathrm{e} \AA\right.$ u.c. $\left.{ }^{-1}\right)$ & 4.78 & 3.72 & 2.74 \\
\hline$P_{\mathrm{b}}\left(\mathrm{e} \AA\right.$ u.c. $\left.{ }^{-1}\right)$ & 0.29 & 0.00 & 0.00 \\
\hline$P_{\mathrm{c}}\left(\mathrm{e} \AA\right.$ u.c. $\left.{ }^{-1}\right)$ & 8.75 & 5.50 & 6.74 \\
\hline$P_{\mathrm{a}}\left(\mathrm{m} C \cdot \mathrm{m}^{-2}\right)$ & 18.0 & 13.9 & 10.0 \\
\hline$P_{\mathrm{b}}\left(\mathrm{m} C \cdot \mathrm{m}^{-2}\right)$ & 1.10 & 0.00 & 0.00 \\
\hline$P_{\mathrm{c}}\left(\mathrm{m} C \cdot \mathrm{m}^{-2}\right)$ & 33.0 & 20.6 & 24.7 \\
\hline
\end{tabular}

We have calculated $\Delta P$ by a density functional approach based on the changes in crystal structures and spin states of (1) (Table 1). We perform these calculations for the three phases: P1 (HS-LS) below $83 \mathrm{~K}, P c$ (HS) between 83 and 250 $\mathrm{K}$, and $C c$ (HS) above $250 \mathrm{~K} .{ }^{41} \mathrm{In}$ order to compare $\Delta P$, the calculations were all performed in the unit cell orientation of the HS Cc phase.

The component of $P$ along the $b$ axis (relative to the HS $C c$ phase) is zero for both HS $C c$ and $P c$ phases while it has a non-zero value of $1.1 \mathrm{mC} \cdot \mathrm{m}^{-2}$ for the HS-LS ordered $P 1$ phase. The calculated value is an order of magnitude larger than the measured value. Such discrepancies are commonly seen in ferroelectric materials and may result from domain formation in the real crystal. In the present case we directly observed the formation of ferroelastic domains with opposite polarization components along the $b$ axis in X-ray diffraction experiments. ${ }^{41}$ Thus if domain-free crystals could be created, even larger ME effects would be expected.

\section{Conductivity during the SCO}

We have investigated the effects of applied electric fields $E$ on the electric polarization. At a constant $T$ and $H=0$, measurements of $P(E)$ did not show any ferroelectric hysteresis for $E$ up to $200 \mathrm{kV} \mathrm{m}^{-1}$. This is very likely due to the fact that switching electric polarization means switching ferroelastic domains. However, we do find conductivity occurring during the SCO. Figure $\mathbf{4 a} \mathbf{a}, \mathbf{b}$ shows the change in charge $\Delta Q$ measured while crossing the SCO as a function of $T$. The quantity $\Delta Q$ is the total charge that flows to the sample's capacitor plates during a measurement, and is usually equal to the electric polarization change $\Delta P$. However here we refer to this data as $\Delta Q$ rather $\Delta P$ because we suspect there is an additional contribution to the total charge from current passing through the sample due to conductivity during the SCO. Figure 4a,b shows $\Delta Q(t)$ in a constant $E$ of 0 , $200 \mathrm{kV} \mathrm{m}^{-1}$, or $-200 \mathrm{kV} \mathrm{m}^{-1}$, measured while $T$ was swept up and down across $T_{1 / 2}$. The $\Delta Q(t)$ plot reflects the expected $\Delta P$ when crossing the SCO, plus an additional component occurring only at the SCO. This second component follows the sign of $E$ and is irreversible, causing $\Delta Q$ at the SCO to climb with each successive $T$ sweep. Since it is unlikely that $\Delta P$ increases continuously to infinity, it is more likely that the sample becomes conducting during the process of the phase transition allowing charge to pass through the sample. We estimate that the resistivity of the mm-sized sample must drop to a few $\mathrm{G} \Omega$ (hundreds of $\mathrm{M} \Omega-\mathrm{cm}$ ) during the SCO for this effect to occur. On the other hand, the samples are robustly insulating with resistances greater than $T \Omega$ and loss tangents of $<1 \times 10^{-5}$ outside the region of the SCO. We were unable to capture the conducting phase by pausing the temperature sweep. 


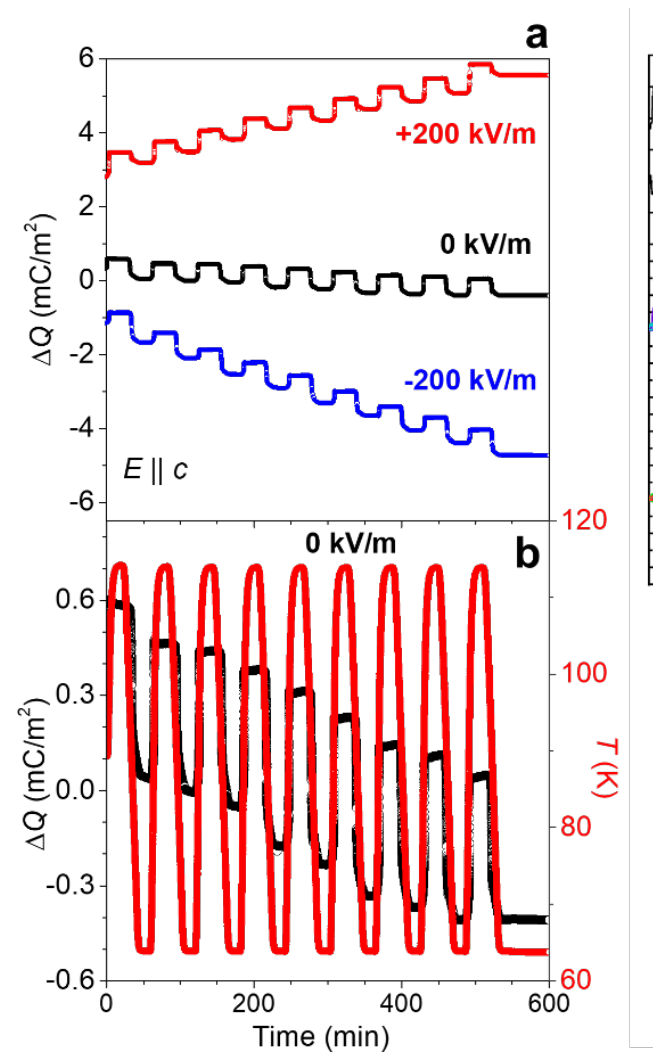

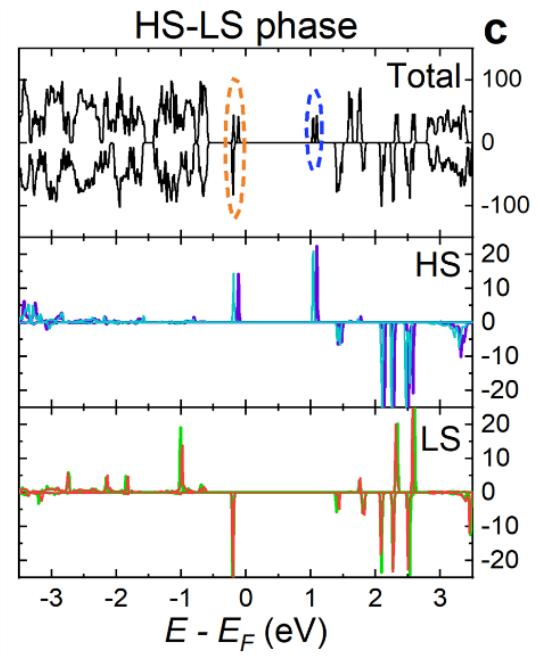

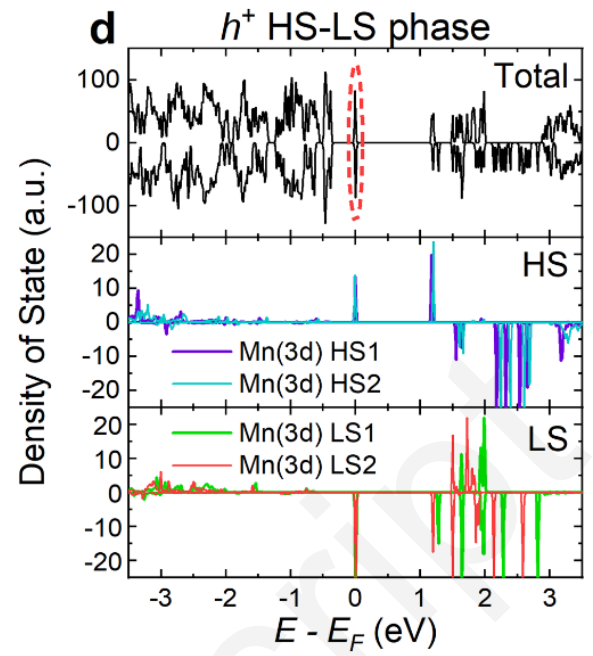

e
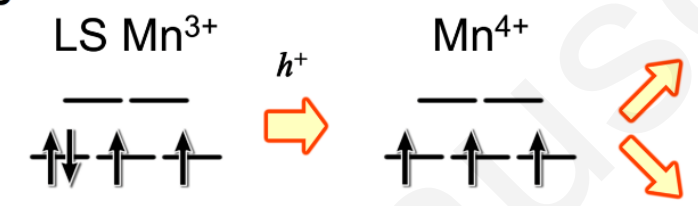

$h^{+}$polaron

at boundaries
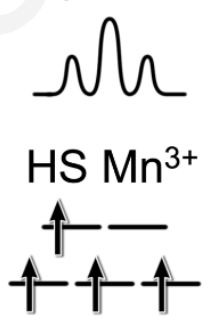

Figure 4. Evidence of conductivity during SCO (1). a, Change in surface charge $\Delta Q$ as the temperature $T$ is swept up and down between 65 and $100 \mathrm{~K}$ across the SCO temperature $T_{1 / 2}$, measured while applying electric fields $E$ of $0,200 \mathrm{kV} \mathrm{m}-1$ and -200 $\mathrm{kV} \mathrm{m}^{-1} . \mathbf{b}, T$ vs time on the right axis and $\Delta Q$ for $E=0$ on the left axis corresponding to the data in a. We interpret $\Delta Q$ as containing two components: a change in electric polarization $\Delta P$ occurring at the SCO phase transition that is of similar size for increasing and decreasing $T$, and a voltage induced current that occurs at the moment of the phase transition. Std. errors are smaller than line widths. c, (upper) the total DOS and projected DOS (PDOS) for (middle) HS and (lower) LS Mn(3d) in the HS-LS ordered phase. Positive and negative values refer to the spin-majority and spin-minority channel, respectively. The dashed orange and blue circles refer to HOMO and LUMO, respectively. Zero energy is the top of HOMO as well as the Fermi energy. d, Same as $\mathbf{c}$ but for the $h^{+}$doped HS-LS ordered phase. The dashed red circles refer to the state at the Fermi level. e. Schematic of how extra charge at the phase boundary can create a polaron.

Thus a likely source of the conductivity is the dynamic phase boundary that forms between the HS-LS ordered phase and the HS phase during the reconstructive $1^{\text {st }}$ order phase transition. This phase boundary is ferroelastic and such phase boundaries between polar phases can often be charged and/or conducting. ${ }^{41,63-65}$

We performed density of states (DOS) calculations to investigate the effect of extra charges at the phase boundary. The extra charge can oxidize or reduce $\mathrm{Mn}^{3+}$ to $\mathrm{Mn}^{4+}$ or $\mathrm{Mn}^{2+}$ respectively depending on the sign of the charge. We focused our calculations on the $\mathrm{Mn}^{3+}$ ions that transition from LS to HS during the SCO. Figure 4c,d show the DOS and the projected density of state (PDOS) of Mn(3d) orbitals in the HS-LS ordered phase and in the HS phase. If the phase boundary is negatively charged, the added electron can only be located at the lowest unoccupied molecular orbital (LUMO). This is the spin-majority Mn(3d) orbitals of the HS state (the blue circle) of which the spin state is unchanged during the phase transition, making negatively charged boundaries trivial. If the boundary is positively charged, the added hole or positive charge can be located at the highest occupied molecular orbital (HOMO), which is the spin-majority $\mathrm{Mn}(3 \mathrm{~d})$ orbital set of the HS or the spin-minority $\mathrm{Mn}(3 \mathrm{~d})$ of the LS (the orange circle).

Figure 4d shows the DOS results of the HS-LS ordered phase with one $h^{+}$per unit cell doped. The total magnetization is $12.5 \mu_{\mathrm{B}}$, namely about $0.75 h^{+}$are doped on spin-minority LS $\mathrm{Mn}^{3+}$ and $0.25 h^{+}$are doped on spin-majority HS $\mathrm{Mn}^{3+}$, so that the $\mathrm{LS} \mathrm{Mn}^{3+}$ has high probability to be activated into $\mathrm{Mn}^{4+}$ with positively charged boundaries. Furthermore, with the doped charge, the HOMO with duplicated degeneracy (the red circle) at each spin channel lies at the Fermi level because there are two LS and two $\mathrm{HS} \mathrm{Mn}^{3+}$ in one unit cell. This does not necessarily mean that a metallic band structure emerges at the charged boundaries. ${ }^{66-67}$ Rather, given the $\mathrm{G} \Omega$ resistance and molecular nature of the crystal we postulate conduction by polaron hopping, which are the quasiparticle carriers in many conductive molecules ${ }^{68-69}$ including SCO materials. ${ }^{70-71}$ After the optimization of the $h^{+}$ doped structure, the splitting of those peaks is only $5 \mathrm{meV}$ for spin-majority and $10 \mathrm{meV}$ for spin-minority, respec- 
tively. This difference between two LS or two HS Mn(3d) orbitals at the Fermi level is tiny. It indicates that one unit cell cannot trap one hole at the measured temperatures, and thus it is more likely that polarons are dynamic with large sizes. During the SCO, the energy from the changing of phonons and the formation of boundaries is large enough to generate polarons. Once a positive polaron is formed, the activated $\mathrm{Mn}^{4+}$ transforms to $\mathrm{HS} \mathrm{Mn}^{3+}$. Figure 4e displays how a LS $\mathrm{Mn}^{3+}$ at the positively charged boundaries become $\mathrm{HS} \mathrm{Mn}^{3+}$ and meanwhile generates one positive polaron in the framework of our theory. The diffusion of polarons can further activate nearby $\mathrm{LS} \mathrm{Mn}^{3+}$, so that it accelerates the SCO with lower energy. To confirm this, we excite one of two LS $\mathrm{Mn}^{3+}$ into the HS by doping with a $h^{+}$. The total energy increases only $8 \mathrm{meV}$. This energy difference is smaller than that of the undoped situation (22 meV) as well as the SCO energy per Mn between the HS-LS ordered and HS phase (31 meV). It indicates that the charged boundaries can reduce the energy required for the SCO.

\section{Conclusion}

In conclusion, we have demonstrated cross coupling between spin state, structure and electric polarization in $\left[\mathrm{Mn}^{\mathrm{III}} \mathrm{L}\right] \mathrm{BPh}_{4}(\mathbf{1})$. Near the SCO critical temperature, we observe magnetic field-induced permanent switching of the spin state, structure and electric polarization due to a $1^{\text {st }}$ order phase transition. The SCO behavior, monitored through the evolution of the HS fraction $\gamma$ from $1 / 2$ to 1 , is driven by a field as low as $8.7 \mathrm{~T}$, which is remarkably low compared to most other magnetic field-induced SCO materials. Due to the $\gamma \eta^{2}$ coupling, the HS state stabilizes the high symmetry phase with $\eta=0$ and consequently suppress the polarization component along the $b$ axis. We also note that conductivity is observed during the SCO, though not outside of it, and likely originates from polaron hopping at the charged phase boundaries forming during the $1^{\text {st }}$ order reconstructive phase transition that accompanies the SCO. $\Delta P(H)$ is $1 / 10$ of the record for any material. ${ }^{52}$ Thus the combination of relatively low magnetic field switching, and giant ME coupling showcases the potential for multifunctionality and applications in SCO materials.

\section{ASSOCIATED CONTENT}

Supporting information is provided with additional structural, magnetic and electric data. This material is available free of charge via the Internet at http://pubs.ac.org.

\section{AUTHOR INFORMATION}

\section{Corresponding Authors}

*vzapf@lanl.gov

*grace.morgan@ucd.ie

\section{Present Addresses}

$\nabla$ The National High Magnetic Field Laboratory, Tallahassee, FL 32310, USA

" Technische Universität Kaiserslautern, Erwin Schrödinger Str. 52, 67663 Kaiserslautern, Germany

$\S$ Idaho National Laboratory, Idaho Falls, ID 83415, USA
\% Physikalisch-Technische Bundesanstalt, Bundesallee 100, 38116 Braunschweig, Germany

\section{Author Contributions}

V.S.Z. led the project. V.S.Z., S.C. and G.G.M. conceived the project. V.B.J. grew the crystals, performed the characterization, analysed a majority of the data in consultation with V.S.Z. J.Y. and H.C. performed calculations. V.S.Z. and S.C. designed the magnetoelectric experiments. S.C., E.D., C.K., and X.D. also performed data collection and analysis. F.W. built and provided a magnetization probe for some of the measurements. E.T. and E.C. collected and analysed the single crystal X-ray diffraction data. V.B.J. and V.S.Z. wrote the manuscript with help from S.C., J.Y., H.C. and E.C.. All authors have given approval to the final version of the manuscript.

\section{Funding Sources}

The scientific work by V.S.Z., H.P.C. and J.Y. was funded by the Center for Molecular Magnetic Quantum Materials (M2QM), an Energy Frontier Research Center funded by the U.S. Department of Energy, Office of Science, Basic Energy Sciences under Award DE SC0019330. G.G.M. would like to thank Science Foundation Ireland (SFI) for support via an Investigator Project Award (19/FFO/6909). V.B.J. was supported by the Irish Research Council GOIPG/2016/73 fellowship. Travel grants for V.B.J.'s research visits to LANL and IPR were funded by Augustinus Fonden (grant no. 18-0338), Oticon Fonden (grant no. 173813), Reinholdt W. Jorck og Hustrus Fond (grant no. 18-JI0573), P.A. Fiskers Fond, A.P. Møller og Hustru Chastine McKinney Møllers Fond til almene Formaal and Christian og Ottilia Brorsons Rejselegat for yngre videnskabsmænd og kvinder. C.T.K was supported by the Irish Research Council GOIPG/2018/2510 and UCD Advance PhD Scheme Supplemental Award. The NHMFL experimental facility at LANL is funded by the U.S. National Science Foundation through Cooperative Grant No. DMR-1157490, the State of Florida, and the U.S. Department of Energy.

\section{ACKNOWLEDGMENT}

The authors acknowledge Michael A. Carpenter, University of Cambridge and Jia Chen, University of Florida for helpful scientific discussions for this paper.

\section{SUPPORTING INFORMATION}

Methods for experimental measurements and discussion of artefacts, additional crystal structure depictions, additional data on magnetization, dielectric constant and electric polarization, and details of the density of states calculations.

\section{REFERENCES}

1. Wang, Y.; Li, J.; Viehland, D., Magnetoelectrics for magnetic sensor applications: status, challenges and perspectives. Mater. Today 2014, 17 (6), 269-275.

2. Heron, J. T.; Schlom, D. G.; Ramesh, R., Electric field control of magnetism using BiFe03-based heterostructures. Appl. Phys. Rev. 2014, 1 (2), 021303.

3. Carman, G. P.; Sun, N., Strain-mediated magnetoelectrics: Turning science fiction into reality. MRS Bull. 2018, 43 (11), 822-828.

4. Pan, C.; Naeemi, A., An Expanded Benchmarking of BeyondCMOS Devices Based on Boolean and Neuromorphic Representative Circuits,. IEEE Journal on Exploratory Solid-State Computational Devices and Circuits 2017, 3, 101.

5. Binek, C.; Doudin, B., Magnetoelectronics with magnetoelectrics. J. Phys.: Condens. Matter 2004, 17, L39. 
6. Bibes, M.; Barthélémy, A., Towards a magnetoelectric memory. Nature Mat. 2008, 7 (6), 425-426.

7. Hu, J.-M.; Nan, C.-W., Opportunities and challenges for magnetoelectric devices. APL Materials 2019, 7 (8), 080905.

$8 . \quad$ Sharma, N.; Bird, J. P.; Binek, C.; Dowben, P. A.; Nikonov, D.; Marshall, A., Evolving magneto-electric device technologies. Semicond. Sci. Technol. 2020, 35, 073001.

9. Fiebig, M.; Lottermoser, T.; Meier, D.; Trassin, M., The evolution of multiferroics. Nature Rev. Mat. 2016, 1 (8), 16046.

10. Spaldin, N. A.; Ramesh, R., Advances in magnetoelectric multiferroics. Nature Mat. 2019, 18 (3), 203-212.

11. Morgan, G. G.; Murnaghan, K. D.; Müller-Bunz, H.; McKee, V.; Harding, C. J., A Manganese(III) Complex That Exhibits Spin Crossover Triggered by Geometric Tuning. Angew. Chem. Int. Ed. 2006, 45 (43), 7192-7195.

12. Martinho, P. N.; Gildea, B.; Harris, M. M.; Lemma, T.; Naik, A. D.; Müller-Bunz, H.; Keyes, T. E.; Garcia, Y.; Morgan, G. G., Cooperative Spin Transition in a Mononuclear Manganese(III) Complex. Angew. Chem. Int. Ed. 2012, 51 (50), 12597-12601.

13. Halcrow, M. A., Spin-crossover Compounds with Wide Thermal Hysteresis. Chem. Lett. 2014, 43 (8), 1178-1188.

14. Pandurangan, K.; Gildea, B.; Murray, C.; Harding, C. J.; MüllerBunz, H.; Morgan, G. G., Lattice Effects on the Spin-Crossover Profile of a Mononuclear Manganese(III) Cation. Chemistry - A European Journal 2012, 18 (7), 2021-2029.

15. Gildea, B.; Harris, M. M.; Gavin, L. C.; Murray, C. A.; Ortin, Y.; Müller-Bunz, H.; Harding, C. J.; Lan, Y.; Powell, A. K.; Morgan, G. G., Substituent Effects on Spin State in a Series of Mononuclear Manganese(III) Complexes with Hexadentate Schiff-Base Ligands. Inorg. Chem. 2014, 53 (12), 6022-6033.

16. Shatruk, M.; Phan, H.; Chrisostomo, B. A.; Suleimenova, A., Symmetry-breaking structural phase transitions in spin crossover complexes. Coord. Chem. Rev. 2015, 289-290, 62-73.

17. Fitzpatrick, A. J.; Trzop, E.; Müller-Bunz, H.; Dîrtu, M. M.; Garcia, Y.; Collet, E.; Morgan, G. G., Electronic vs. structural ordering in a manganese(iii) spin crossover complex. Chem. Comm. 2015, 51 (99), 17540-17543.

18. Senthil Kumar, K.; Ruben, M., Emerging trends in spin crossover (SCO) based functional materials and devices. Coord. Chem. Rev. 2017, 346, 176-205.

19. Brooker, S., Spin crossover with thermal hysteresis: practicalities and lessons learnt. Chem. Soc. Rev. 2015, 44 (10), 28802892.

20. Mason, H. E.; Li, W.; Carpenter, M. A.; Hamilton, M. L.; Howard, J. A. K.; Sparkes, H. A., Structural and spectroscopic characterisation of the spin crossover in [Fe(abpt)2(NCS)2] polymorph A. New J. Chem. 2016, 40 (3), 2466-2478.

21. Ortega-Villar, N.; Muñoz, M. C.; Real, J. A., Symmetry Breaking in Iron(II) Spin-Crossover Molecular Crystals. Magnetochemistry 2016, $2(1), 16$.

22. Collet, E.; Guionneau, P., Structural analysis of spincrossover materials: From molecules to materials. Comptes Rendus Chimie 2018, 21 (12), 1133-1151.

23. Nakano, M.; Matsubayashi, G.-e.; Matsuo, T., Dielectric behavior of manganese(III) spin-crossover complex [Mn(taa)]. Phys. Rev. B 2002, 66 (21), 212412.

24. Chikara, S.; Gu, J.; Zhang, X. G.; Cheng, H.-P.; Smythe, N.; Singleton, J.; Scott, B.; Krenkel, E.; Eckert, J.; Zapf, V. S., Magnetoelectric behavior via a spin state transition. Nature Comm. 2019, 10 (1), 4043. 25. Otsuki, Y.; Kimura, S.; Awaji, S.; Nakano, M., Magnetocapacitance effect and magnetostriction by the field-induced spin-crossover in [MnIII(taa)]. AIP Advances 2019, 9 (8), 085219.

26. Lefter, C.; Tan, R.; Dugay, J.; Tricard, S.; Molnár, G.; Salmon, L.; Carrey, J.; Nicolazzi, W.; Rotaru, A.; Bousseksou, A., Unidirectional electric field-induced spin-state switching in spin crossover based microelectronic devices. Chem. Phys. Lett. 2016, 644, 138-141.

27. Zhang, X.; Palamarciuc, T.; Létard, J.-F.; Rosa, P.; Lozada, E. V.; Torres, F.; Rosa, L. G.; Doudin, B.; Dowben, P. A., The spin state of a molecular adsorbate driven by the ferroelectric substrate polarization. Chem. Comm. 2014, 50 (18), 2255-2257.
28. Jornet-Mollá, V.; Duan, Y.; Giménez-Saiz, C.; Tang, Y.-Y.; Li, P.F.; Romero, F. M.; Xiong, R.-G., A Ferroelectric Iron(II) Spin Crossover Material. Angew. Chem. Int. Ed. 2017, 56 (45), 14052-14056.

29. Qi, Y.; Müller, E. W.; Spiering, H.; Gütlich, P., The effect of a magnetic field on the high-spin $\alpha$ low-spin transition in [Fe(phen)2(NCS)2]. Chem. Phys. Lett. 1983, 101 (4), 503-505.

30. Lejay, J.; Jansen, A. G. M.; Wyder, P.; Bronger, W.; Kläui, W., Spin equilibrium of $\mathrm{Co} 3+$ complexes influenced by a magnetic field. Phys. Rev. B 1991, 43 (10), 8196-8198.

31. Garcia, Y.; Kahn, O.; Ader, J.-P.; Buzdin, A.; Meurdesoif, Y.; Guillot, M., The effect of a magnetic field on the inversion temperature of a spin crossover compound revisited. Phys. Lett. A 2000, 271 (1), 145-154.

32. Negre, N.; Goiran, M.; Bousseksou, A.; Haasnoot, J.; Boukheddaden, K.; Askenazy, S.; Varret, F., High magnetic field induced spin transition, H.M.F.I.S.T. effect, in [Fe0.52Ni0.48(btr)2(NCS)2]H2O. Synth. Met. 2000, 115 (1), 289-292.

33. Bousseksou, A.; Negre, N.; Goiran, M.; Salmon, L.; Tuchagues, J.-P.; Boillot, M.-L.; Boukheddaden, K.; Varret, F., Dynamic triggering of a spin-transition by a pulsed magnetic field. Eur. Phys. J. B 2000, 13 (3), 451-456.

34. Kimura, S.; Narumi, Y.; Kindo, K.; Nakano, M.; Matsubayashi, G.-e., Field-induced spin-crossover transition of [MnIII(taa)] studied under pulsed magnetic fields. Phys. Rev. B 2005, 72 (6), 064448.

35. Altarawneh, M. M.; Chern, G. W.; Harrison, N.; Batista, C. D.; Uchida, A.; Jaime, M.; Rickel, D. G.; Crooker, S. A.; Mielke, C. H.; Betts, J. B.; Mitchell, J. F.; Hoch, M. J. R., Cascade of Magnetic Field Induced Spin Transitions in LaCo03. Phys. Rev. Lett. 2012, 109 (3), 037201.

$36 . \quad$ Ridier, K.; Bas, A.-C.; Zhang, Y.; Routaboul, L.; Salmon, L.; Molnár, G.; Bergaud, C.; Bousseksou, A., Unprecedented switching endurance affords for high-resolution surface temperature mapping using a spin-crossover film. Nature Comm. 2020, 11, 3611.

$37 . \quad$ Ridier, K.; Bas, A. C.; Shalabaeva, V.; Nicolazzi, W.; Salmon, L.; Molnár, G.; Bousseksou, A.; Lorenc, M.; Bertoni, R.; Collet, E.; Cailleau, H., Finite Size Effects on the Switching Dynamics of Spin-Crossover Thin Films Photoexcited by a Femtosecond Laser Pulse. Adv. Mater. 2019, 31, 1901361.

38. $\quad$ Piedrahita-Bello, M.; Martin, B.; Salmon, L.; Molnár, G.; Demont, P.; Bousseksou, A., Mechano-electric coupling in P(VDF$\mathrm{TrFE}$ )/spin crossover composites $\dagger$

J. Mat. Chem. C 2020, 6042.

39. Mosey, A.; Dale, A. S.; Hao, G.; N’Diaye, A.; Dowben, P. A.; Cheng, R., Quantitative Study of the Energy Changes in VoltageControlled Spin Crossover Molecular Thin Films

J. Phys. Chem. Lett. 2020, 11, 8231.

40. Weber, B.; Dowben, P., Preface to the JPCM Special Issue on Molecular Magnetism. J. Physics: Condensed Matter 2020, 32, 440201.

41. Jakobsen, V. B.; Trzop, E.; Gavin, L. C.; Dobbelaar, E.; Chikara, S.; Ding, X.; Esien, K.; Müller-Bunz, H.; Felton, S.; Zapf, V. S.; Collet, E.; Carpenter, M. A.; Morgan, G. G., Stress-Induced Domain Wall Motion in a Ferroelastic Mn3+ Spin Crossover Complex. Angew. Chem. Int. Ed. 2020, 59 (32), 13305-13312.

42. Molnár, G.; Rat, S.; Salmon, L.; Nicolazzi, W.; Bousseksou, A., Spin Crossover Nanomaterials: From Fundamental Concepts to Devices. Adv. Mater. 2018, 30 (5), 1703862.

43. Létard, J.-F., Photomagnetism of iron(ii) spin crossover complexes-the T(LIESST) approach. J. Mater. Chem. 2006, 16 (26), $2550-2559$.

44. Sato, O., Dynamic molecular crystals with switchable physical properties. Nature Chem. 2016, 8 (7), 644-656.

45. Chastanet, G.; Lorenc, M.; Bertoni, R.; Desplanches, C., Lightinduced spin crossover-Solution and solid-state processes. Comptes Rendus Chimie 2018, 21 (12), 1075-1094.

46. Zapf, V. S.; Sengupta, P.; Batista, C. D.; Nasreen, F.; WolffFabris, F.; Paduan-Filho, A., Magnetoelectric effects in an organometallic quantum magnet. Phys. Rev. B 2011, 83 (14), 140405.

47. Pardo, E.; Train, C.; Liu, H.; Chamoreau, L.-M.; Dkhil, B.; Boubekeur, K.; Lloret, F.; Nakatani, K.; Tokoro, H.; Ohkoshi, S.-i.; Verdaguer, M., Multiferroics by Rational Design: Implementing 
Ferroelectricity in Molecule-Based Magnets. Angew. Chem. Int. Ed. 2012, 51 (33), 8356-8360.

$48 . \quad$ Tian, Y.; Stroppa, A.; Chai, Y.; Yan, L.; Wang, S.; Barone, P.; Picozzi, S.; Sun, Y., Cross coupling between electric and magnetic orders in a multiferroic metal-organic framework. Sci. Rep. 2014, 4, 6062.

49. Mun, E.; Weickert, F.; Kim, J.; Scott, B. L.; Miclea, C. F.; Movshovich, R.; Wilcox, J.; Manson, J.; Zapf, V. S., Partially disordered antiferromagnetism and multiferroic behavior in a frustrated Ising system CoCl2-2SC(NH2)2. Phys. Rev. B 2016, 93 (10), 104407.

50. Gómez-Aguirre, L. C.; Pato-Doldán, B.; Mira, J.; Castro-García, S.; Señarís-Rodríguez, M. A.; Sánchez-Andújar, M.; Singleton, J.; Zapf, V. S., Magnetic Ordering-Induced Multiferroic Behavior in [CH3NH3][Co(HCOO)3] Metal-Organic Framework. J. Am. Chem. Soc. 2016, 138 (4), 1122-1125.

51. Jain, P.; Stroppa, A.; Nabok, D.; Marino, A.; Rubano, A.; Paparo, D.; Matsubara, M.; Nakotte, H.; Fiebig, M.; Picozzi, S.; Choi, E. S.; Cheetham, A. K.; Draxl, C.; Dalal, N. S.; Zapf, V. S., Switchable electric polarization and ferroelectric domains in a metal-organic-framework. npj Quantum Mater. 2016, 1, 16012.

52. Aoyama, T.; Yamauchi, K.; Iyama, A.; Picozzi, S.; Shimizu, K.; Kimura, T., Giant spin-driven ferroelectric polarization in $\mathrm{TbMnO3}$ under high pressure. Nature Comm. 2014, 5 (1), 4927.

53. Miyashita, S.; Konishi, Y.; Tokoro, H.; Nishino, M.; Boukheddaden, K.; Varret, F., Structures of Metastable States in Phase Transitions with a High-Spin Low-Spin Degree of Freedom. Prog. Theor. Phys. 2005, 114 (4), 719-735.

54. Varret, F.; Slimani, A.; Boukheddaden, K.; Chong, C.; Mishra, H.; Collet, E.; Haasnoot, J.; Pillet, S., The propagation of the thermal spin transition of [Fe(btr)2(NCS)2]·H2O single crystals, observed by optical microscopy. New J. Chem. 2011, 35 (10), 2333-2340.

55. Slimani, A.; Varret, F.; Boukheddaden, K.; Garrot, D.; Oubouchou, H.; Kaizaki, S., Velocity of the High-Spin Low-Spin Interface Inside the Thermal Hysteresis Loop of a Spin-Crossover Crystal, via Photothermal Control of the Interface Motion. Phys. Rev. Lett. 2013, 110 (8), 087208.

56. $\quad$ Sy, M.; Varret, F.; Boukheddaden, K.; Bouchez, G.; Marrot, J.; Kawata, S.; Kaizaki, S., Structure-Driven Orientation of the High-SpinLow-Spin Interface in a Spin-Crossover Single Crystal. Angew. Chem. 2014, 126 (29), 7669-7672.

57. Paez-Espejo, M.; Sy, M.; Boukheddaden, K., Elastic Frustration Causing Two-Step and Multistep Transitions in SpinCrossover Solids: Emergence of Complex Antiferroelastic Structures. J. Am. Chem. Soc. 2016, 138 (9), 3202-3210.

58. Traiche, R.; Sy, M.; Oubouchou, H.; Bouchez, G.; Varret, F.; Boukheddaden, K., Spatiotemporal Observation and Modeling of Remarkable Temperature Scan Rate Effects on the Thermal Hysteresis in a Spin-Crossover Single Crystal. J. Phys. Chem. C 2017, 121 (21), 11700-11708.

59. Fourati, H.; Milin, E.; Slimani, A.; Chastanet, G.; Abid, Y.; Triki, S.; Boukheddaden, K., Interplay between a crystal's shape and spatiotemporal dynamics in a spin transition material. Phys. Chem. Chem. Phys. 2018, 20 (15), 10142-10154.

60. Affes, K.; Fourati, H.; Slimani, A.; Boukheddaden, K., Effects of High-Spin-Low-Spin Lattice Misfit on the Nucleation and Propagation Velocities of Elastic Interfaces in Cooperative Spin-Crossover Solids. J. Phys. Soc. Jpn. 2019, 88 (12), 124701.

61. Chernyshov, D.; Bürgi, H.-B.; Hostettler, M.; Törnroos, K. W., Landau theory for spin transition and ordering phenomena in Fe(II) compounds. Phys. Rev. B 2004, 70 (9), 094116.

62. Watanabe, H.; Tanaka, K.; Bréfuel, N.; Cailleau, H.; Létard, J.F.; Ravy, S.; Fertey, P.; Nishino, M.; Miyashita, S.; Collet, E., Ordering phenomena of high-spin/low-spin states in stepwise spin-crossover materials described by the ANNNI model. Phys. Rev. B 2016, 93 (1), 014419.

63. Jia, C.-L.; Mi, S.-B.; Urban, K.; Vrejoiu, I.; Alexe, M.; Hesse, D., Atomic-scale study of electric dipoles near charged and uncharged domain walls in ferroelectric films. Nature Mat. 2008, 7 (1), 57-61.

64. Li, L.; Gao, P.; Nelson, C. T.; Jokisaari, J. R.; Zhang, Y.; Kim, S.J.; Melville, A.; Adamo, C.; Schlom, D. G.; Pan, X., Atomic Scale Structure Changes Induced by Charged Domain Walls in Ferroelectric Materials. Nano Letters 2013, 13 (11), 5218-5223.

65. Bednyakov, P. S.; Sturman, B. I.; Sluka, T.; Tagantsev, A. K.; Yudin, P. V., Physics and applications of charged domain walls. npj Comp. Mat. 2018, 4 (1), 65.

66. Ohtomo, A.; Hwang, H. Y., A high-mobility electron gas at the LaAlO3/SrTiO3 heterointerface. Nature 2004, 427 (6973), 423-426.

67. Nakagawa, N.; Hwang, H. Y.; Muller, D. A., Why some interfaces cannot be sharp. Nature Mat. 2006, 5 (3), 204-209.

$68 . \quad$ Galperin, M.; Ratner, M. A.; Nitzan, A., Hysteresis, Switching, and Negative Differential Resistance in Molecular Junctions: A Polaron Model. Nano Letters 2005, 5 (1), 125-130.

69. Punk, M.; Dumitrescu, P. T.; Zwerger, W., Polaron-tomolecule transition in a strongly imbalanced Fermi gas. Phys. Rev. A 2009, 80 (5), 053605.

$70 . \quad$ Rotaru, A.; Gural'skiy, I. y. A.; Molnár, G.; Salmon, L.; Demont, P.; Bousseksou, A., Spin state dependence of electrical conductivity of spin crossover materials. Chem. Comm. 2012, 48 (35), 4163-4165.

71. Lefter, C.; Davesne, V.; Salmon, L.; Molnár, G.; Demont, P.; Rotaru, A.; Bousseksou, A., Charge Transport and Electrical Properties of Spin Crossover Materials: Towards Nanoelectronic and Spintronic Devices. Magnetochemistry 2016, 2 (1), 18. 
Giant magnetoelectric coupling is observed in a $\mathrm{Mn}^{3+}$ spin crossover complex, $\left[\mathrm{Mn}^{\mathrm{II}} \mathrm{L}\right] \mathrm{BPh}{ }_{4}\left(\mathrm{~L}=(3,5-\mathrm{diBr} \text {-sal })_{2} 323\right)$. Magnetic fields induce both a spin crossover and a structural phase transition between different polar space groups. This giant magnetoelectric effect is moreover hysteretic - once switched by fields the spin state and electric polarization change remains trapped.

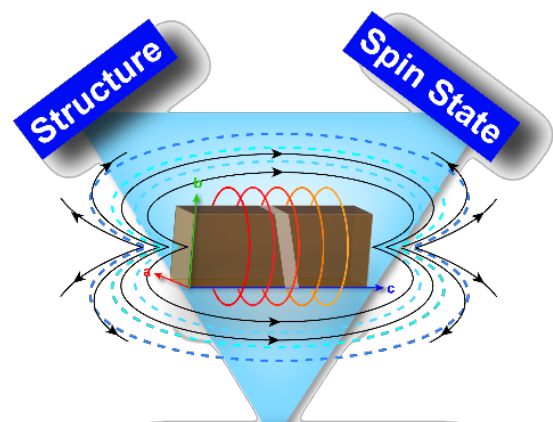

Electric Properties 


\section{Supporting Information}

\section{Giant Magnetoelectric Coupling and Magnetic Field-Induced Permanent Switching in a Spin Crossover Mn(III) Complex}

\section{Table of contents}

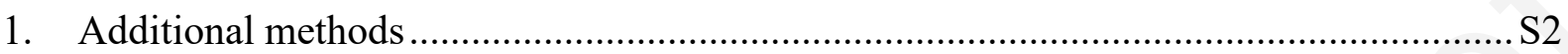

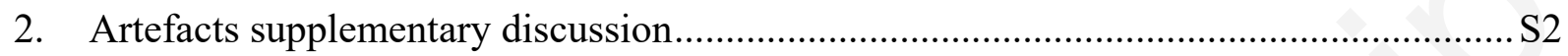

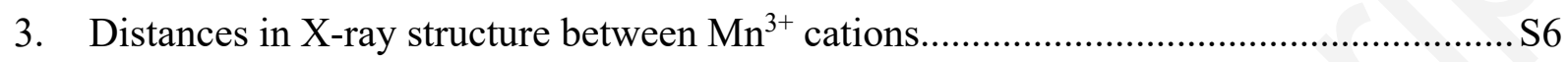

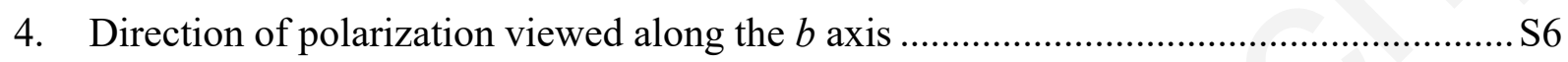

5. Direction of polarization viewed along [101] direction.............................................. S7

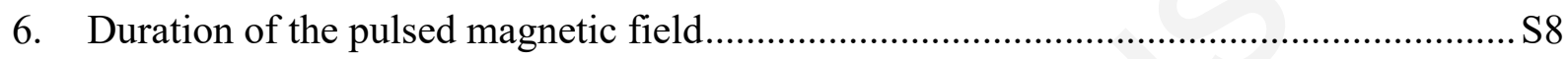

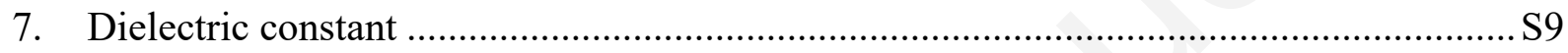

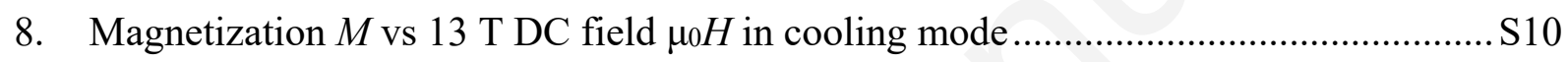

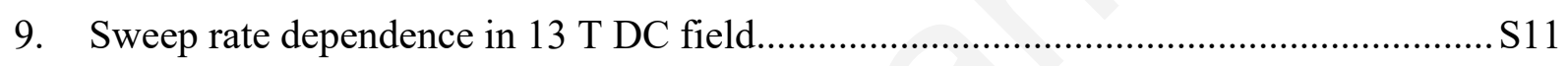

10. Magnetization $M$ vs $60 \mathrm{~T}$ pulsed field $\mu_{0} H$ in warming mode .................................... S13

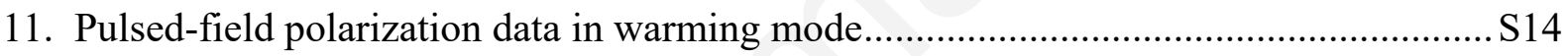

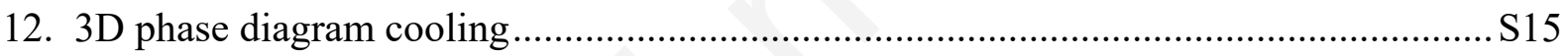

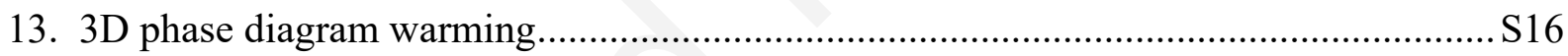

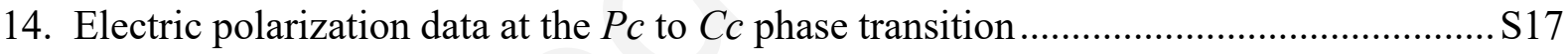

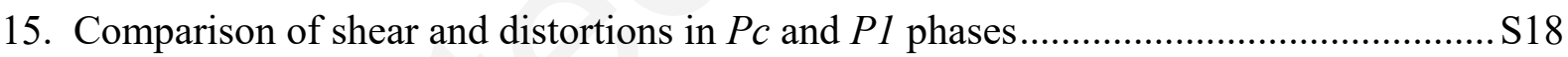

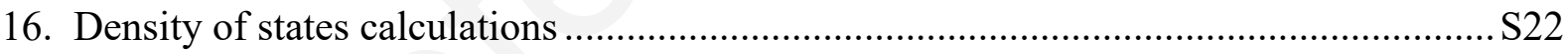

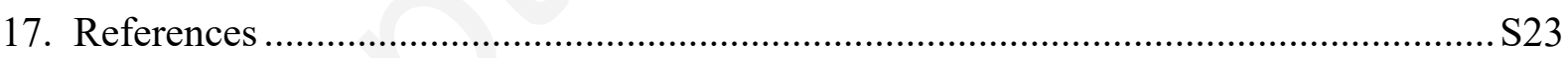




\section{Additional methods}

Sample Preparation: The details of preparation of single crystals of $\left[\mathrm{Mn}^{\mathrm{III}} \mathrm{L}\right] \mathrm{BPh} 4(\mathbf{1})$ are given here. ${ }^{1}$

$D C$ and pulsed magnetic field measurements: $M$ was measured in a Vibrating Sample Magnetometer (VSM) in a 13 T Physical Properties Measurement System (PPMS) by Quantum Design. In the same PPMS, a custom probe with coaxial cables was used to measure electric polarization and conductivity across capacitor plates applied to the $a b$-faces of a single crystal using silver paint. ${ }^{2-4}$ Measurements along other axes yielded much smaller $\Delta P(H) . H \| c$ and $H$ $\perp c$ gave the same results. The dielectric loss tangent of the crystals was $<1 \cdot 10^{-5}$ at $70 \mathrm{~K}$, away from the spin crossover. $\Delta P(T, H)$ or $\Delta Q(T, H)$ was determined using a Keithley $6517 \mathrm{~A}$ electrometer. Dielectric constant was measured with an Andeen-Hagerling 2500A capacitance bridge at $30 \mathrm{~V}$ and $1 \mathrm{kHz}$.

Pulsed field measurements of the magnetization and electric polarization were performed in capacitor-driven resistive magnets up to $65 \mathrm{~T}$ with a few milliseconds rise time. The magnetic field vs time is shown in Figure S4. The pulsed field magnetization was determined by an inductive technique in compensated coils ${ }^{5}$ with sample-in sample-out background subtraction. $\Delta P(H)$ was determined by recording the $H$-induced change in surface charge with a Stanford Research $570 .^{2-3}$ Where not otherwise marked, measurements were conducted on one single crystal for magnetic and electric fields along the $c$ axis. $T$ was controlled using ${ }^{4} \mathrm{He}$ gas or liquid via a Cernox thermometer mounted near the magnetization sample or on sapphire together with the polarization sample. Throughout, standard errors are smaller than linewidths and point size when not shown.

DFT calculations: First-principles calculations were carried out using density functional theory with the projector augmented wave potential ${ }^{6}$ as implemented in the Vienna ab-initio simulation package (VASP) ${ }^{7-8}$ The plane-wave functions, expanded with an energy cutoff of $600 \mathrm{eV}$, were employed throughout calculations. The exchange-correlation energy was described by the generalized gradient approximation in the Perdew, Burke, and Ernzerhof form. ${ }^{9}$ The spin states from the experiment were used. DFT-D3 method with van der Waals correction ${ }^{10}$ was employed for structure optimization and all forces on each atom were converged to $10 \mathrm{meV} \AA^{-1}$. The polarization vectors were obtained by the evaluation of the Berry phase expressions. ${ }^{11}$ For electronic structure calculations, we employed plus Hubbard $U$ method $^{12}$ with $U=3.0 \mathrm{eV}, J=$ $0.9 \mathrm{eV}$ on $\mathrm{Mn}(3 \mathrm{~d})$ orbitals to describe $3 d$ electrons of Mn. The k points are sampled on a $\Gamma$ centered $3 \times 2 \times 3$ mesh.

\section{Artefacts supplementary discussion}

Conductivity artefacts: The millimeter-sized single crystals showed loss tangents less than $1 \times 10^{-5}$ measured by a capacitor bridge at $1 \mathrm{kHz}$. Thus conductivity artefacts in the measurements can be ruled out. A momentary change in conductivity during the spin crossover is described in the main paper.

Voltage artefacts: In general there can be expected to be voltage across the sample at all times due to Schottky barriers between the capacitor plates and the sample and/or thermoelectric 
effects in measurement wires. Differences in the Schottky barriers of the two capacitor plates on either size of the sample cause a net voltage of a few to tens of $\mathrm{mV}$. This can be observed e.g. in Figure 4 where the " 0 voltage" data is consistent with a small net voltage.

Dilatometry artefacts: Another artefact to consider is the fact that the sample changes volume by around half a percent during the spin crossover transition where we measure the change in electric polarization. This means that the distance between the capacitor plates and the surface area of the sample will change. If there is a voltage across the sample, this length change could produce a change in the capacitance, and thus a change in the measured electric polarization. Qualitatively we can rule out such an artefact because 1) it would scale with the voltage across the sample, and 2) it would be reversible in temperature since the length change is the same when traversing the SCO in both directions. We did not observe such a signal that is reversible and scales with voltage.

However, a quantitative argument to rule out such a dilatometry artefact is given below. A typical sample has a dielectric constant $\varepsilon$ near 3 , a capacitance $C$ of $1.3 \times 10^{-14} \mathrm{~F}$ with a $0.5 \mathrm{~mm}^{2}$ surface area $A$ of the capacitor plate, a $0.5 \mathrm{~mm}$ distance between plates $d$, and a $0.28 \%$ change in $d$ at the spin crossover. Given the equation for capacitance of a parallel plate capacitor, and the largest voltage applied in our experiments of $100 \mathrm{~V}$ e.g. in Figure 4, we can calculate the change in charge on the capacitor plates due to a $0.28 \%$ change in distance between capacitor plates, $\delta \mathrm{d} / \mathrm{d}=.0028$

$$
\begin{aligned}
& C=\frac{Q}{V}=\frac{\varepsilon \cdot A}{d} \\
& C+\delta C=\frac{Q}{V} \cdot(1+\delta Q / Q) \cong \frac{\varepsilon \cdot A}{d} \cdot(1-\delta d / d) \\
& \frac{\delta Q}{Q}=\left(\frac{-\delta d}{d}\right) \\
& \delta Q=-Q \frac{\delta d}{d}=-\left(\frac{\varepsilon \cdot A \cdot V}{d}\right)\left(\frac{\delta d}{d}\right)= \\
& -\left(\frac{\left(3 \cdot \frac{8.85 \times 10^{-12} F}{m}\right)\left(0.5 \cdot 10^{-6} m^{2}\right) \cdot(100 \mathrm{~V})}{0.5 \cdot 10^{-3} \mathrm{~m}}\right)(.0028)=-7.4 \cdot 10^{-15} C
\end{aligned}
$$

Per unit surface area:

$$
\frac{\delta Q}{A}=\left(\frac{-7.4 \cdot 10^{-15} C}{0.5 \cdot 10^{-3} m^{2}}\right)=-14.8 \cdot 10^{-9} \frac{m C}{m^{2}}
$$

This dilatometry artefact is eight orders of magnitude smaller than the measured changes in electric polarization and can thus be safely neglected. A similar calculation shows that changes in the surface area $A$ of the plates cause negligibly small artefacts. 


\section{Pulsed field artefacts}

\section{Magnetization backgrounds}

The magnetization is recorded by a coil that experiences an induced voltage as the magnetic field through the coil changes. The magnetic field can be caused by the external $65 \mathrm{~T}$ magnet or by the sample itself. The coil is counterwound to exclude the mostly spatially uniform $65 \mathrm{~T}$ magnetic field - e.g. the inner windings are wound clockwise and the outer windings are anticlockwise. The coil is designed such that a spatially uniform magnetic field will cause minimal signal in the coil. On the other hand, the sample located inside the coil does not create a spatially uniform magnetic field, and thus its signal will be different in the inner clockwise windings compared to the outer anti-clockwise windings, and a net signal will occur in the coil. The background of the external $65 \mathrm{~T}$ magnetic field is subtracted in a three step process: 1) The magnetization pickup coil is counterwound. 2) Since the counterwinding is not perfect, especially as the coil thermally contracts at low temperatures, the signal from an additional single turn of the coil is measured and a fraction of it is subtracted by an electronic circuit, such that the background with the sample outside of the coil is zero. 3) The coil is measured once for the sample in the coil and once for the sample outside the coil. The sample can be moved in and out of the coil without warming the system.

Additional backgrounds are caused by vibration of the magnetometer during the sudden millisecond magnetic field pulse. These backgrounds cause oscillations that are noted in the figure caption of the main paper.

\section{Electric polarization backgrounds}

In millisecond pulsed magnetic fields, backgrounds of the electric polarization were measured separately and found to be negligibly small compared to the sample's measured electric polarization.

\section{Heating or cooling}

A thermometer is mounted near the sample for magnetization measurements and on a sapphire plate together with the sample for electric polarization measurements. Additional thermalization is provided by helium gas. The thermometer records the temperature before each magnetic field pulse. During a magnetic field pulse, heating can occur due to eddy currents in metallic objects. We find this heating to be negligible in polarization and magnetization measurements.

Heating can also occur due to magnetocaloric effects - we find this to be usually negligible but it can be significant when unusually entropic events take place such as spin crossover behavior. Since there is very little time for the sample to exchange heat with its substrate, it is in a quasiadiabatic thermal situation. Thus entropy changes in the sample's magnetic properties can cause heating or cooling.

This effect is negligibly small in the HS-LS ordered state or the HS state, however the entropy change is more significant at the spin crossover, which can cause cooling as the sample transitions from the HS-LS ordered state to the HS state. ${ }^{[1]}$ In addition, domain motion and physical motion of the sample changing shape during the first order phase transition can cause irreversible heating. However, since the temperature changes do not happen until the spin 
crossover occurs, we can accurately find the magnetic field where the spin crossover onsets on upsweep.

\section{DC field artefacts}

In DC fields, measurements are conducted slowly enough $\left(1 \mathrm{~K} \mathrm{~min}^{-1}\right)$ for the sample to remain at constant temperature, and the temperature is monitored continuously during the measurements in both electric polarization and magnetization measurements. Backgrounds for magnetization and electric polarization measurements are negligible or subtracted.

\section{Substrate strain effects}

One question that arises is whether the MIESST effect in our measurements is intrinsic, or whether it results from strain effects since the sample is affixed to a substrate. The sample undergoes a volume change of about $0.5 \%$ and length changes of $1.1 \%, 1.4 \%$ and $0.28 \%$ along the $a, b$ and $c$ axes respectively at the SCO referenced to the $C c$ unit cell. ${ }^{[1]}$ One could speculate that the fact that the sample's length is physically constrained on one surface by a sample holder could introduce hysteresis and prevent the sample from returning to the HS-LS state after the magnetic field is removed. The electric polarization sample is a single crystal with dimensions between 0.5 and $2 \mathrm{~mm}$ that are glued down on one surface with GE 7031 varnish. The magnetization samples consist of several smaller samples affixed to a sample holder with Apiezon N grease or GE 7031 varnish and are thus potentially constrained on all surfaces. We rule out this hypothesis by measuring the magnetization of single crystals that were loosely placed in a capsule without any grease or glue. We find that the MIESST effect can be observed both for glued down and loose crystals, showing that it is an intrinsic effect. 


\section{Distances in $\mathrm{X}$-ray structure between $\mathrm{Mn}^{3+}$ cations}
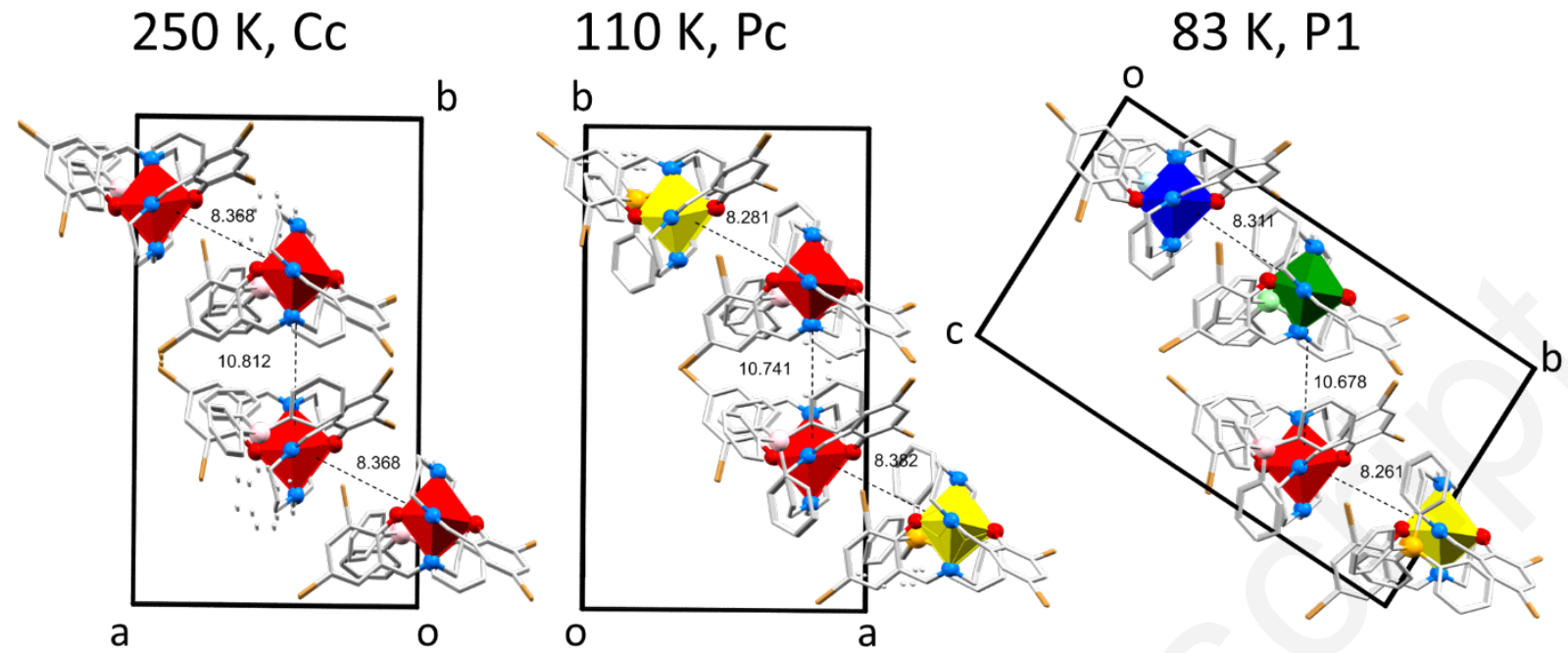

Figure S1. Intermolecular distance between $\mathrm{Mn}^{3+}$ complex cations in the three different phases of (1) showing the increase (blue and green $\mathrm{LS} \mathrm{Mn}^{3+}$ complex cations) and decrease in distances (green LS and red HS Mn ${ }^{3+}$ complex, and red and yellow HS $\mathrm{Mn}^{3+}$ complex cations) in the $P 1$ phases compared to the $P c$ and $C c$ phase.

\section{Direction of polarization viewed along the $b$ axis}

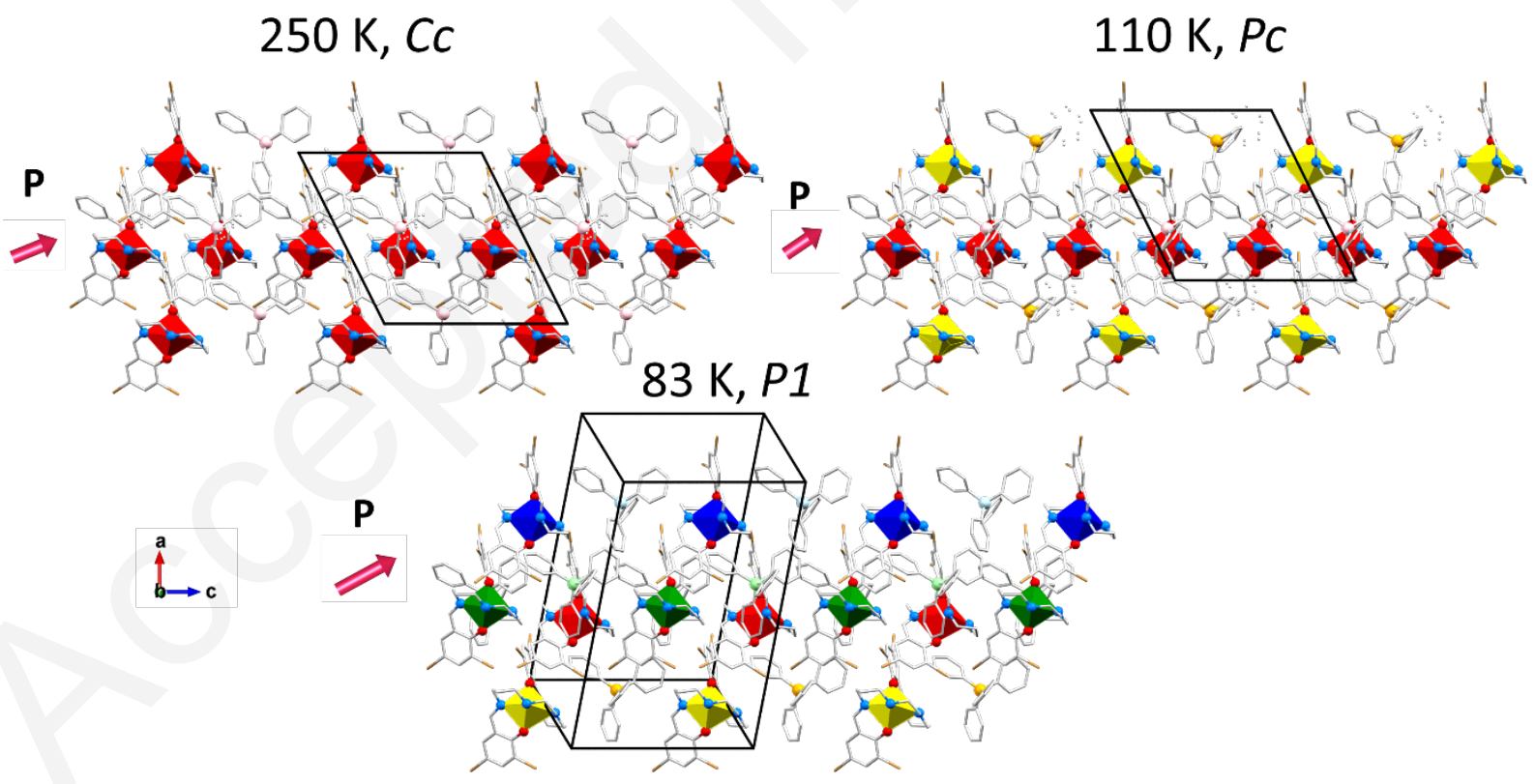

Figure S2. Structure and packing of $\left[\mathrm{Mn}^{\mathrm{III}} \mathrm{L}\right] \mathrm{BPh} 4(\mathbf{1})$ viewed along the $b$ axis for the HS $C c$ cell with the other two structures orientated accordingly. The directions of bulk electric polarization are shown on the left side of each subfigure. 


\section{Direction of polarization viewed along [101] direction}

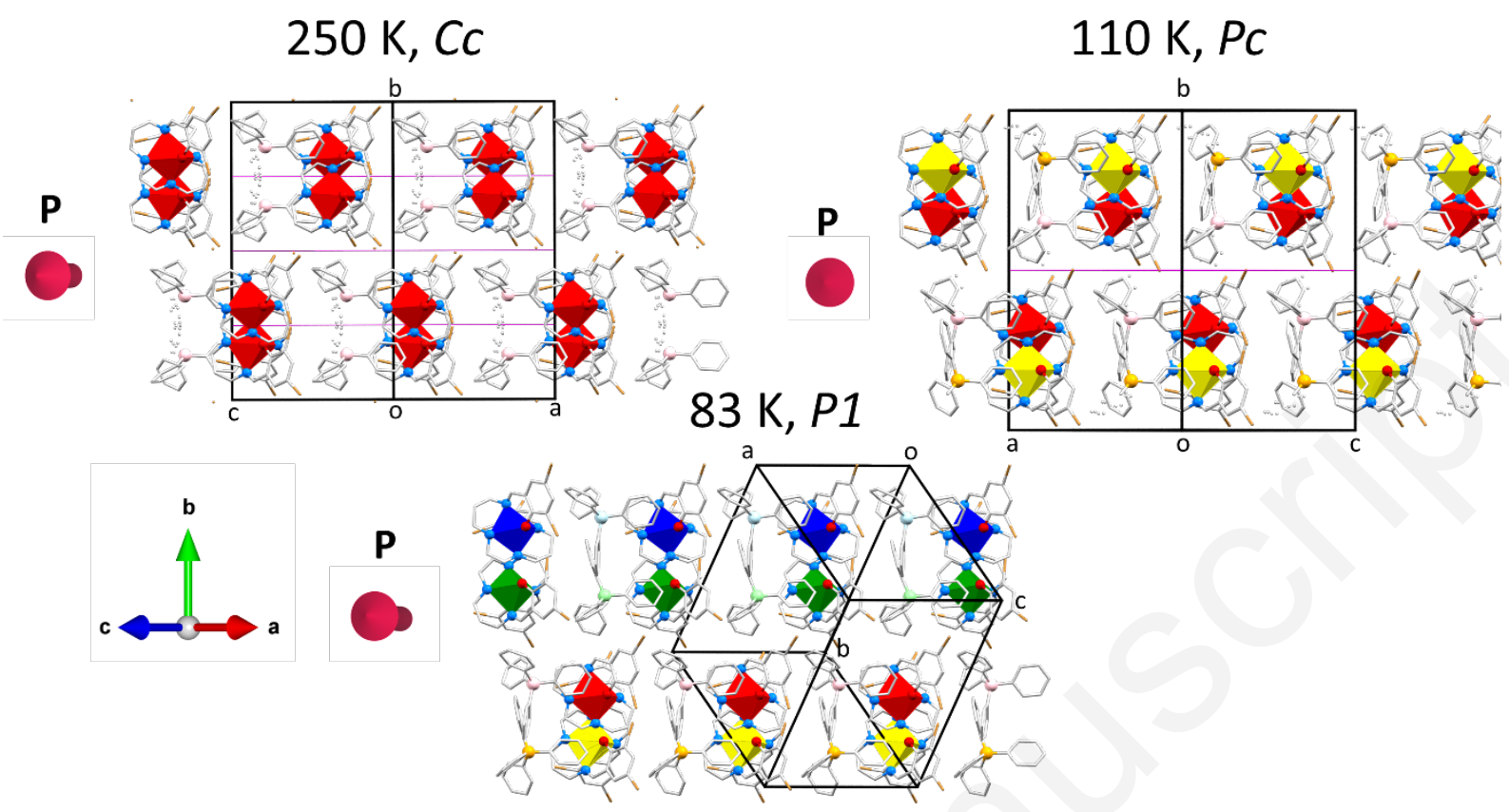

Figure S3. Structure and packing of $\left[\mathrm{Mn}^{\mathrm{III}} \mathrm{L}\right] \mathrm{BPh}_{4}(\mathbf{1})$ viewed along the [101] direction for the HS $C c$ cell with the other two structures orientated accordingly. The purple planes in the structures indicate the glide planes in the $C c$ and $P c$ structure which are lost in the $P 1$ structure. The directions of bulk electric polarization are shown on the left side of each subfigure. 


\section{Duration of the pulsed magnetic field}

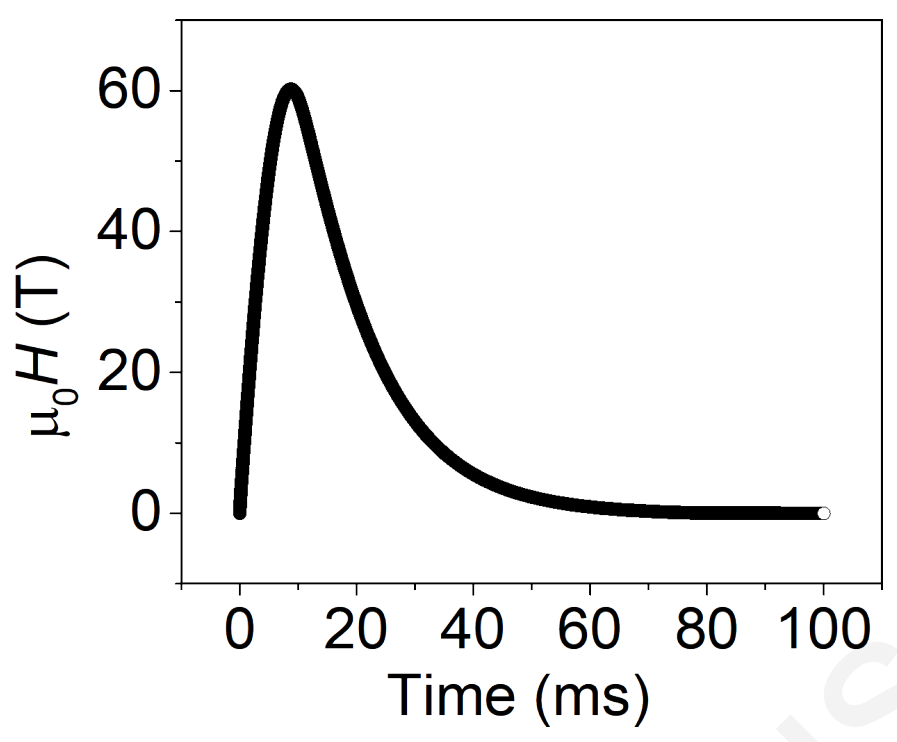

Figure S4. The pulsed magnetic field in a $60 \mathrm{~T}$ pulsed field magnet plotted as magnetic field, $\mu_{0} H(\mathrm{~T})$ vs time (ms). The magnetic field is measured by the voltage induced in a copper coil and calibrated to a known standard, or to characteristic quantum oscillations that occur at high fields in copper. 


\section{Dielectric constant}
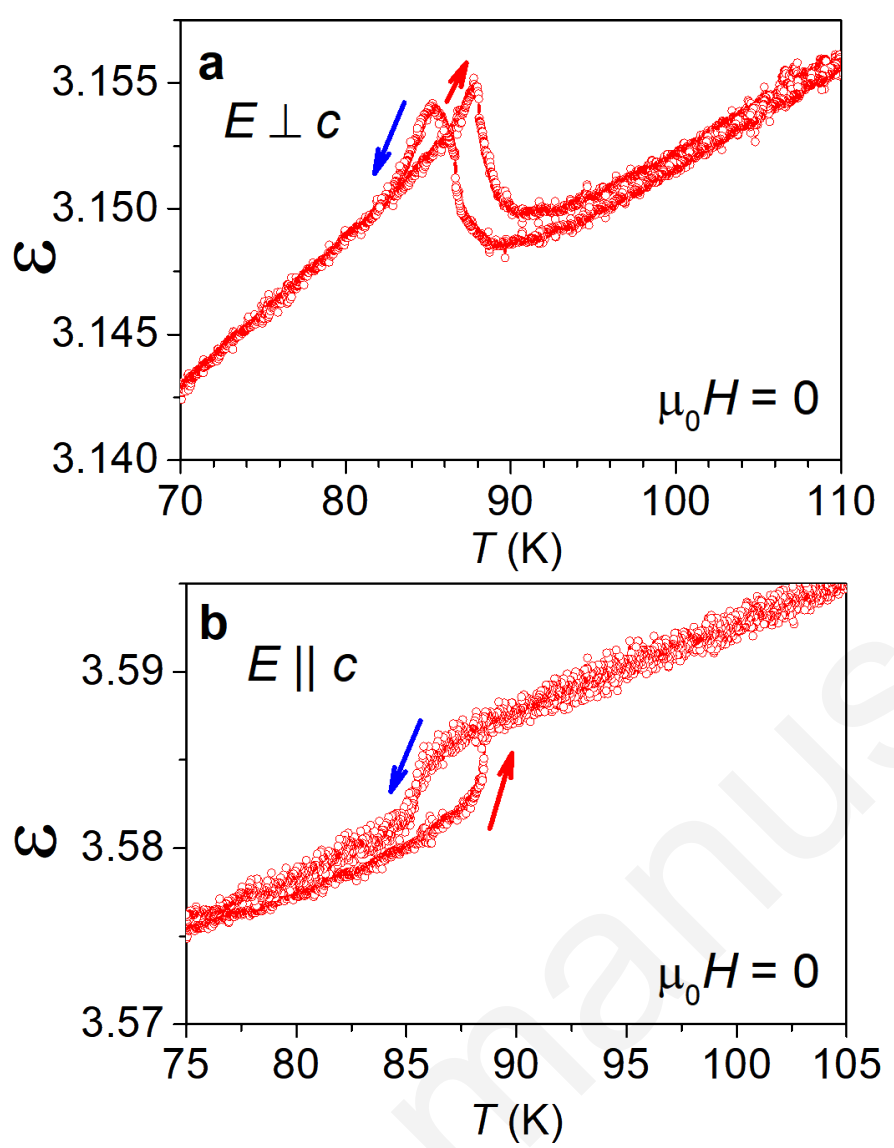

Figure S5. The dielectric constant $\varepsilon$ as a function of temperature $T$ measured at zero field, $H=$ 0, for the electric field in $E \perp H$ and $E \| H$ configurations measured on two different single crystals. The arrows indicate the direction of the temperature sweep. 


\section{Magnetization $M$ vs $13 \mathrm{~T}$ DC field $\mu_{0} H$ in cooling mode}

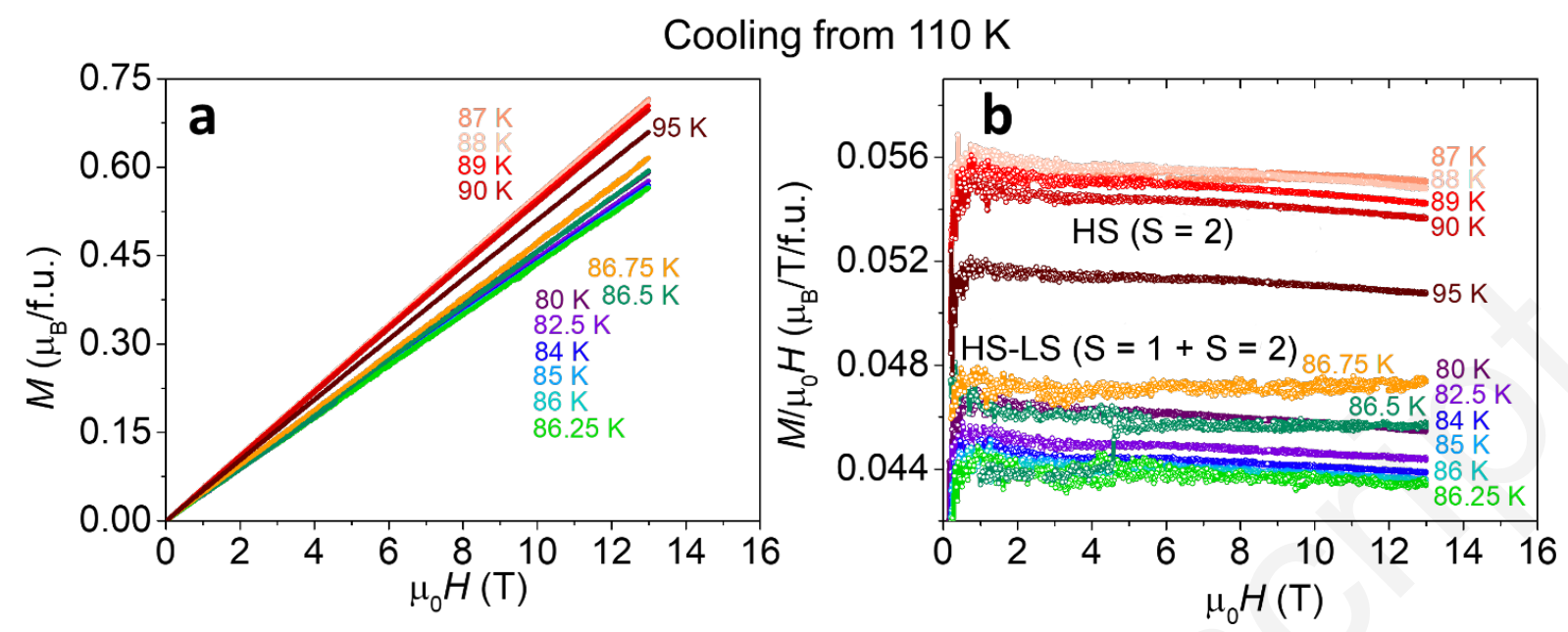

Figure S6. Magnetization $M$ vs magnetic field $\mu_{0} H$ measurements for fields swept from 0 to 13 $\mathrm{T}$ and back to zero. Data is measured with a history of cooling from $110 \mathrm{~K}$ to the desired $T$. a, $M\left(\mu_{0} H\right)$ measured with a history of warming for different $T$ as shown and $\mathbf{b}$, the same data plotted as $M / \mu_{0} H$ to better distinguish the different spin states. Data is shown for $T$ above and below the thermal spin crossover temperature. We label the HS-LS $(1: 1 \mathrm{~S}=1$ and $\mathrm{S}=2)$ and the HS $(\mathrm{S}=2)$ regions. 


\section{Sweep rate dependence in $13 \mathrm{~T}$ DC field}

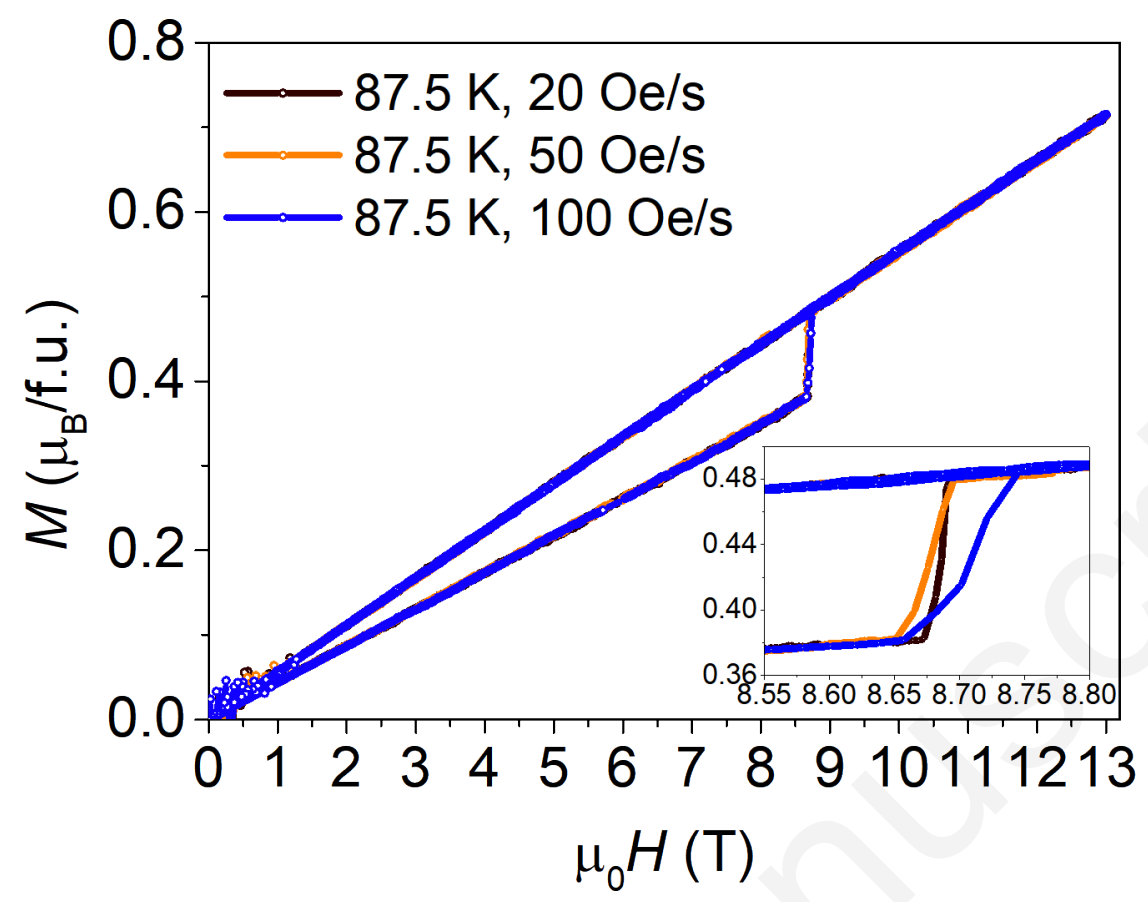

Figure S7. The field-induced switching effect shown at different sweep rates of the magnetic field, $\mu_{0} H$ plotted as magnetization $M$ versus magnetic field, $\mu_{0} H$. The data was collected at $T$ $=87.5 \mathrm{~K}$ and at sweep rates of $2 \mathrm{mT} \mathrm{s}^{-1}$ (black line), $5 \mathrm{mT} \mathrm{s}^{-1}$ (orange line) and $10 \mathrm{mT} \mathrm{s}^{-1}$ (blue line). This effect is observed between $8.65 \mathrm{~T}$ and $8.7 \mathrm{~T}$ for all sweep rates. Sweep rate dependence is expected at first order phase transitions as discussed in the main text, since the field sweep rate competes with the speed of domain nucleation and growth. 


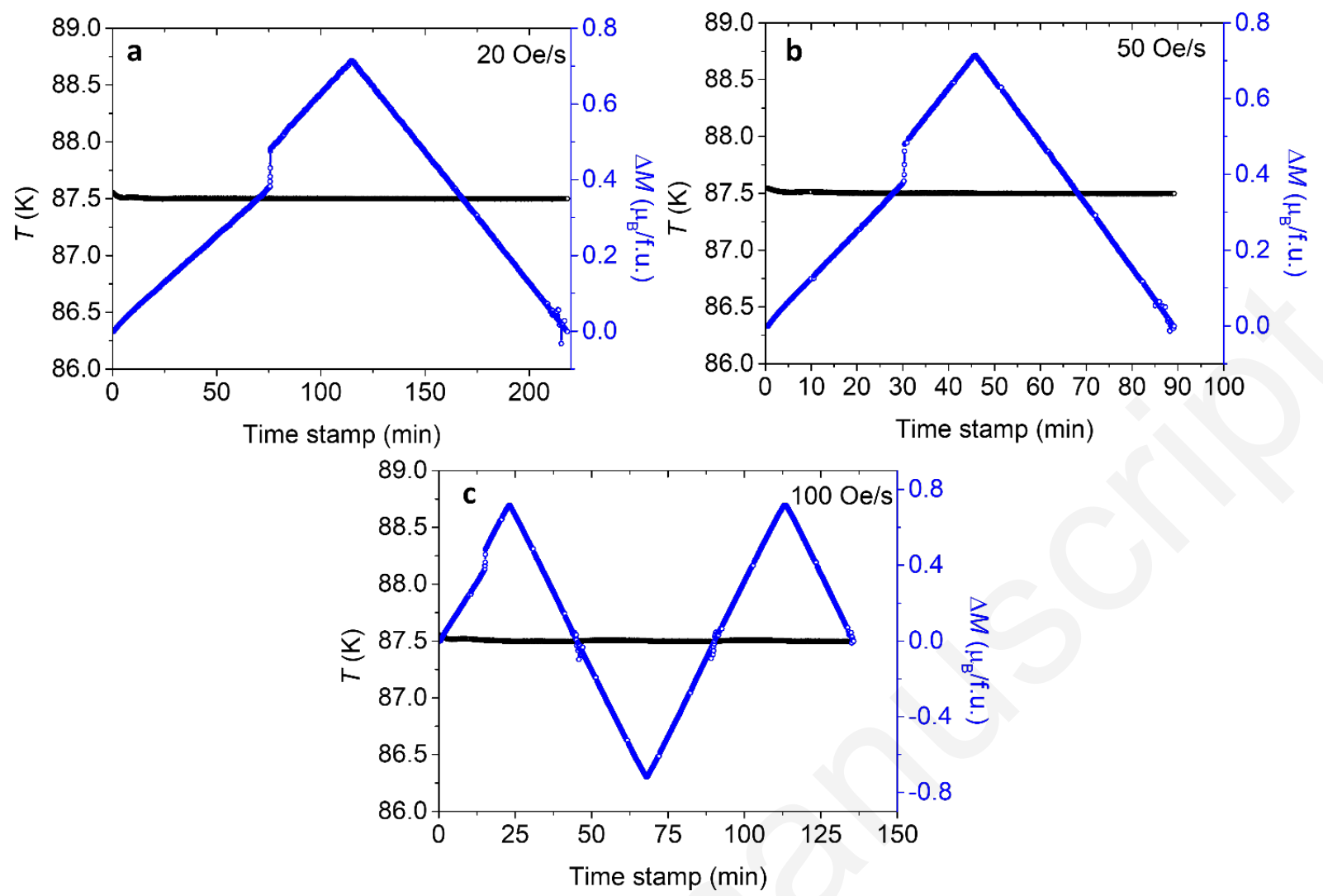

Figure S8. Field-induced switching effect shown as changes in magnetization $M$ as a function of time for different sweep rates of a. $2 \mathrm{mT} \mathrm{s}^{-1}$, b. $5 \mathrm{mT} \mathrm{s}^{-1}$ and c. $10 \mathrm{mT} \mathrm{s}^{-1}$, blue curves. The temperature versus time was monitored during the measurements to ensure it was stable when applying the magnetic field to the sample. 


\section{Magnetization $M$ vs $60 \mathrm{~T}$ pulsed field $\mu_{0} H$ in warming mode}

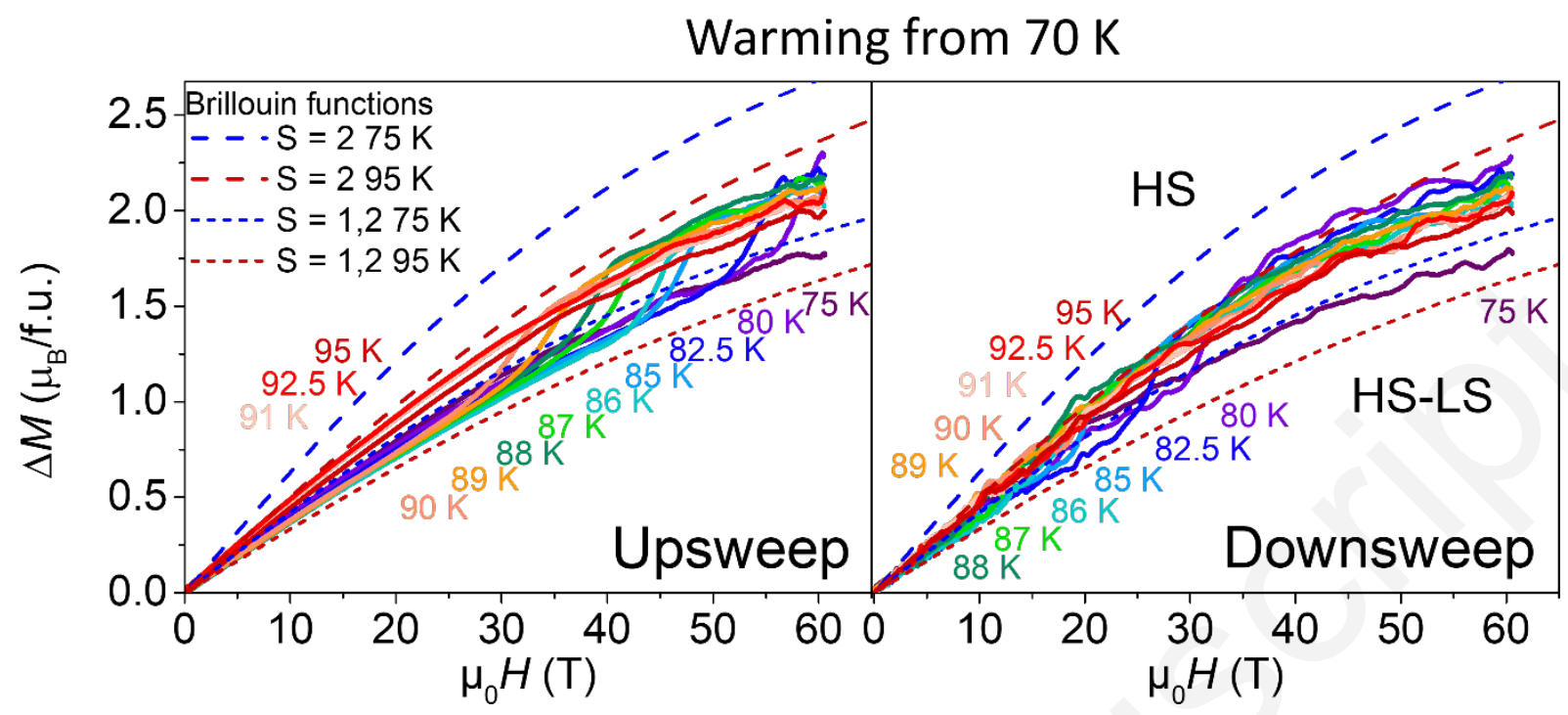

Figure S9. Magnetization $M$ vs magnetic field $\mu_{0} H$ measured in millisecond pulsed magnetic fields up to $60 \mathrm{~T}$. The data is taken on a. rising field while b. shows falling field data. The latter data is noisier since the magnetic field sweep rate is slower on falling and thus there is less signal and more time for mechanical oscillations. The data is taken after warming from $70 \mathrm{~K}$ to the measurement $T$. Blue/red dashed lines are Brillouin functions for the HS states $(\mathrm{S}=2)$, with the upper and lower lines spanning the measured temperatures as shown in the legend. Similarly, short dashed lines show the Brillouin functions for HS-LS ordered state (mixture of $\mathrm{S}=1$ and $\mathrm{S}=2)$. 


\section{Pulsed-field polarization data in warming mode}

\section{Tesla}

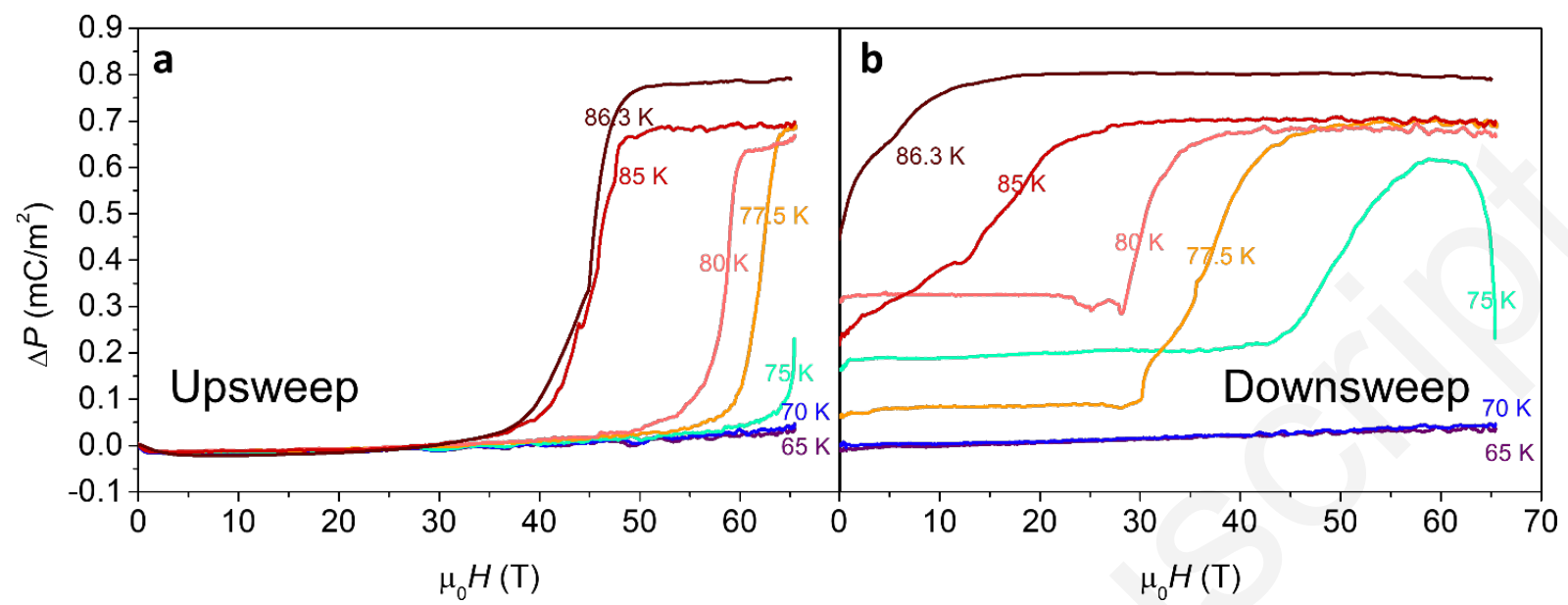

Figure S10. Change in electric polarization $\Delta P$ vs magnetic field $\mu_{0} H$ in a $65 \mathrm{~T}$ pulsed field magnet with maximum field of $65 \mathrm{~T}$ used for a. rising field at different temperatures $T$ as indicated. b. Same as a but for falling field at different temperatures $T$ as indicated. Data is measured after a temperature history of heating from $70 \mathrm{~K}$ to the final measurement temperature between every magnetic field pulse. 


\section{3D phase diagram cooling}

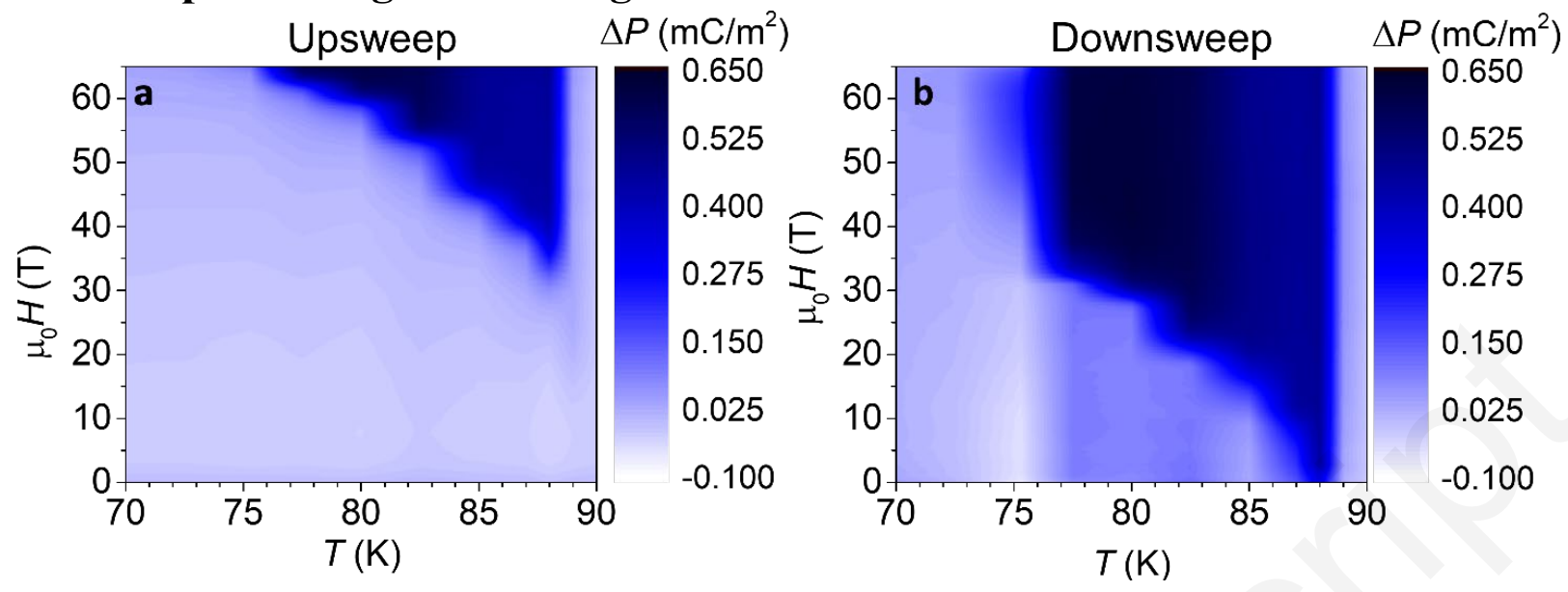

Figure S11. Magnetic field, $\mu_{0} H$ vs temperature, $T 3 \mathrm{D}$ contour phase diagram of [ $\left.\mathrm{Mn}^{\mathrm{III}} \mathrm{L}\right] \mathrm{BPh} 4$ (1) for $E \| H$ in pulsed magnetic fields for a. rising field (upsweep) and b. falling field (downsweep) up to $65 \mathrm{~T}$. The blue color-mapped regions show the spontaneous electric polarization. Data is measured after a temperature history of cooling from $110 \mathrm{~K}$ to the final measurement temperature between every magnetic field pulse. 


\section{3D phase diagram warming}

\section{Tesla}

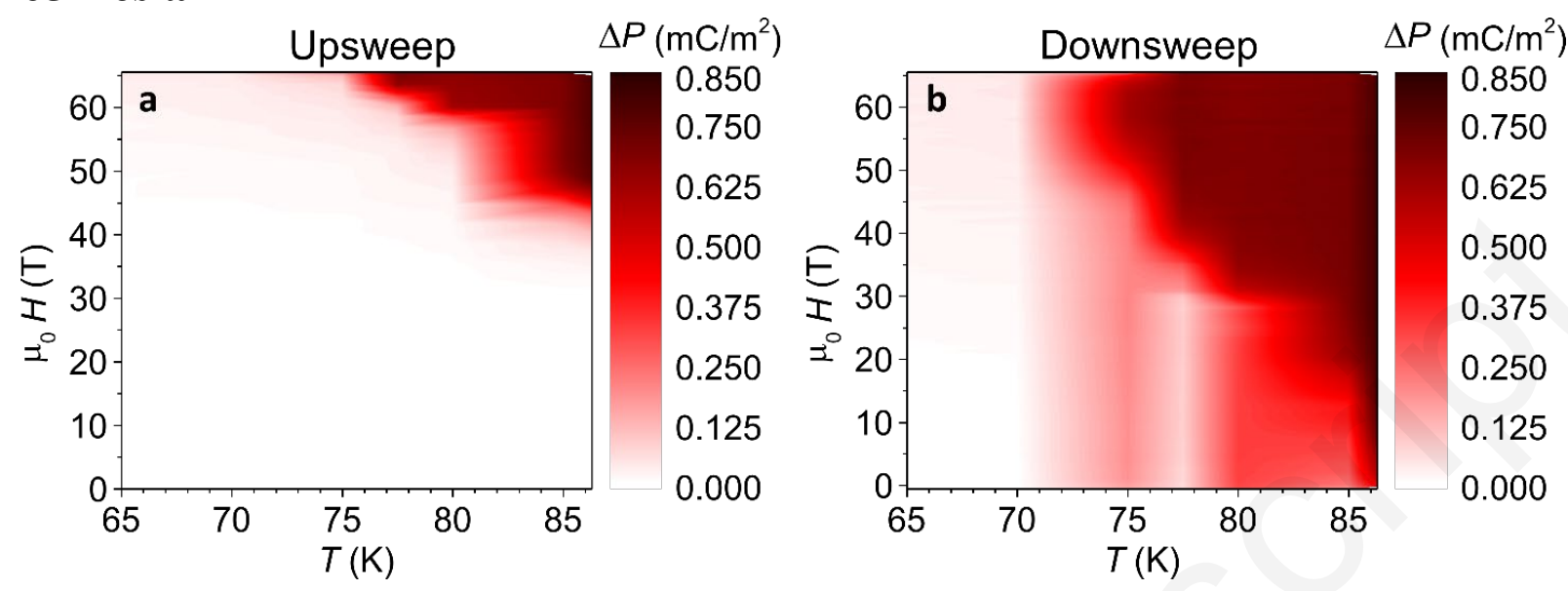

Figure S12. Magnetic field, $\mu_{0} H$ vs temperature, $T$ 3D contour phase diagram of $\left[\mathrm{Mn}^{\mathrm{III}} \mathrm{L}\right] \mathrm{BPh} 4$ (1) for $E \| H$ in pulsed magnetic fields for rising field (upsweep) and falling field (downsweep) up to $65 \mathrm{~T}$. The red color-mapped regions show the spontaneous electric polarization. Data is measured after a temperature history of warming from $70 \mathrm{~K}$ to the final measurement temperature between every magnetic field pulse. 
14. Electric polarization data at the $P c$ to $C c$ phase

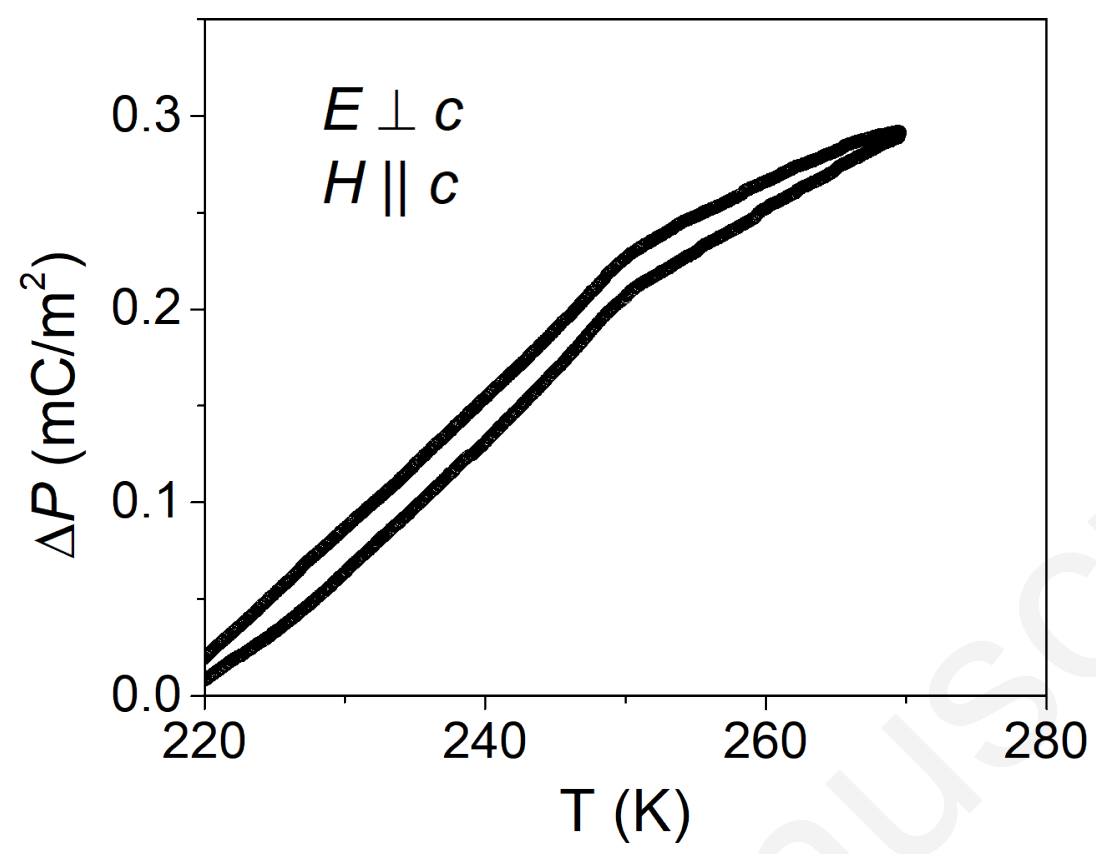

Figure S13. Electric polarization vs temperature measured perpendicular to the $c$ axis of the crystal, for the magnetic field parallel to the $c$ axis. The second order structural phase transition between the $P c$ and $C c$ phases near $250 \mathrm{~K}$ produces a change in slope of the electric polarization. Std. errors are smaller than line widths. 


\section{Comparison of shear and distortions in $P c$ and $P 1$ phases}

Pc

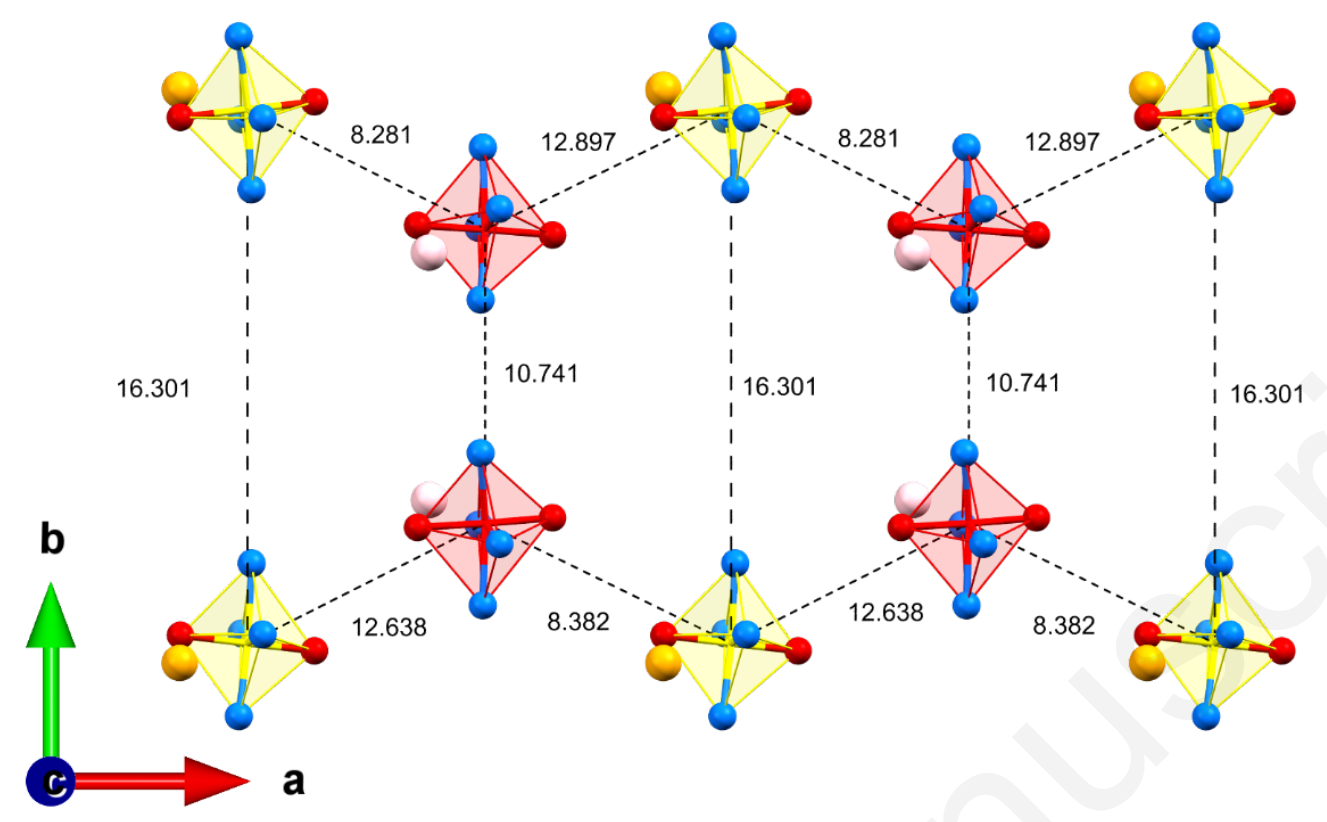

P1

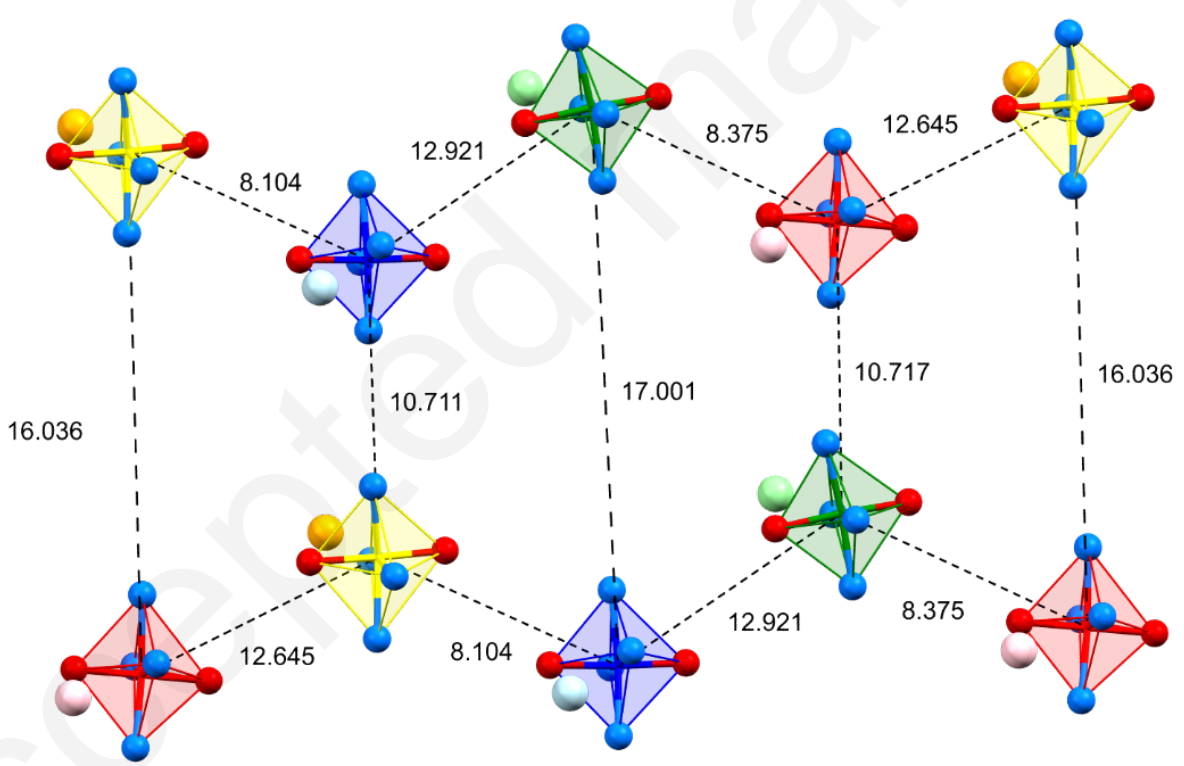

Figure S14. Change in intermolecular $\mathrm{Mn} \cdots \mathrm{Mn}$ distances, viewed along the $c$ axis of the Pc unit cell, between the $\mathrm{Mn}^{3+}$ complex cations in the $P c$ and $P 1$ phases shown as black dashed lines between colored polyhedrons (red, $\mathrm{S}=2$, HS; yellow, $\mathrm{S}=2$, HS; blue, $\mathrm{S}=1$, LS; green, $\mathrm{S}=1$, LS). Only the $\mathrm{N}_{4} \mathrm{O}_{2}{ }^{2-}$ donor atoms around the $\mathrm{Mn}^{3+}$ center is shown. Only the $\mathrm{B}$ of the $\mathrm{BPh}_{4}{ }^{-}$counteranion is shown with all atoms other than the boron atom removed for clarity. The SCO-induced decreases in bond lengths between the $\mathrm{N}_{\text {amine }}$ and $\mathrm{N}_{\text {imine }}$ donor atoms and the $\mathrm{Mn}^{3+}$ center are one of the major sources of distortion at this phase transition. The distances are shown between adjacent $\mathrm{Mn}^{3+}$ cations along the $a$ axis and between each row along the $b$ axis of the $P c$ unit cell. 


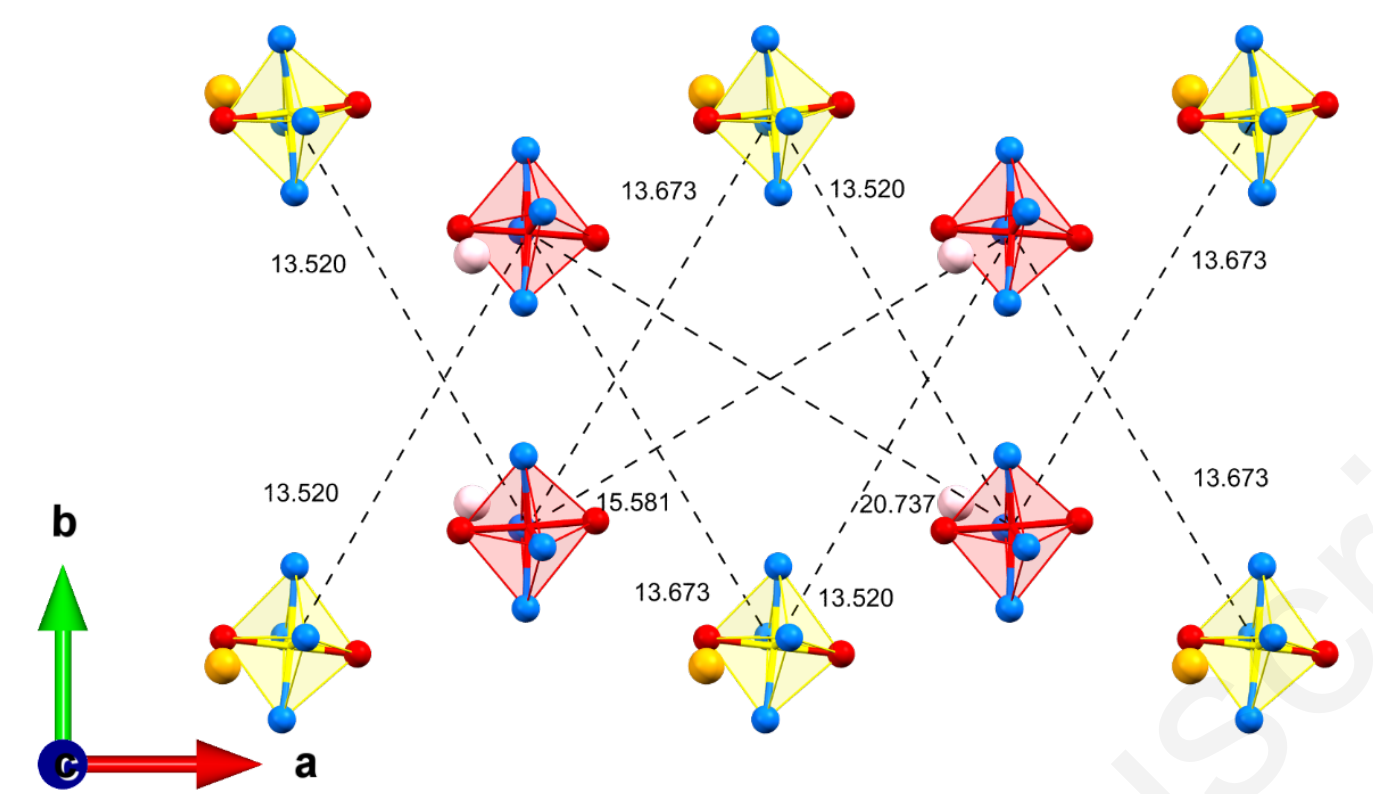

\section{$P 1$}

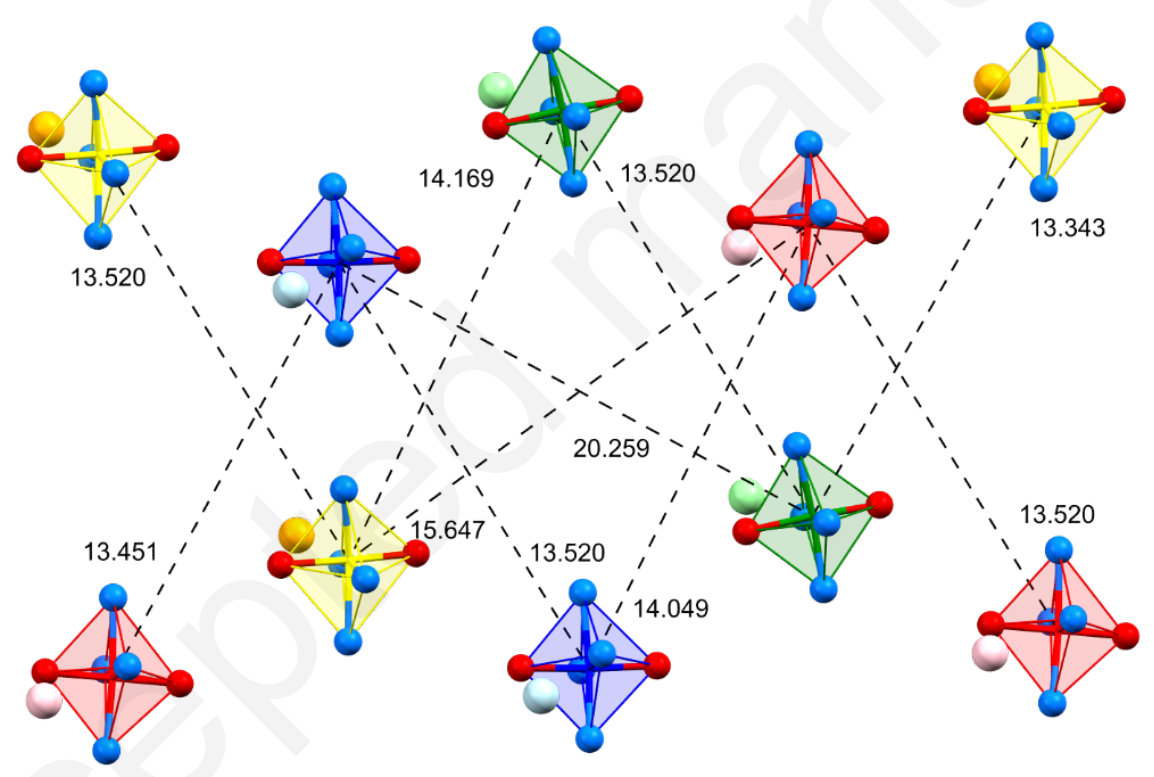

Figure S15. Change in intermolecular $\mathrm{Mn} \cdots \mathrm{Mn}$ distances, viewed along the $c$ axis, between the $\mathrm{Mn}^{3+}$ complex cations in the $P c$ and $P 1$ phases shown as black dashed lines between colored polyhedrons (red, $\mathrm{S}=2$, HS; yellow, $\mathrm{S}=2$, HS; blue, $\mathrm{S}=1$, LS; green, $\mathrm{S}=1$, LS). Only the $\mathrm{N}_{4} \mathrm{O}_{2}{ }^{2-}$ donor atoms around the $\mathrm{Mn}^{3+}$ center is shown. The boron atom of the $\mathrm{BPh}_{4}^{-}$ counteranion is shown as balls) while all atoms other than the boron in $\mathrm{BPh}_{4}{ }^{-}$are removed for clarity. The SCO causes significant distortions due to decrease in bond lengths of the $\mathrm{N}_{\text {amine }}$ and $\mathrm{Nimine}$ donor atoms to the $\mathrm{Mn}^{3+}$ center. Representative distances showing the distortions from the $P 1$ to the $P c$ structure are indicated with black dashed lines. 
$P c$

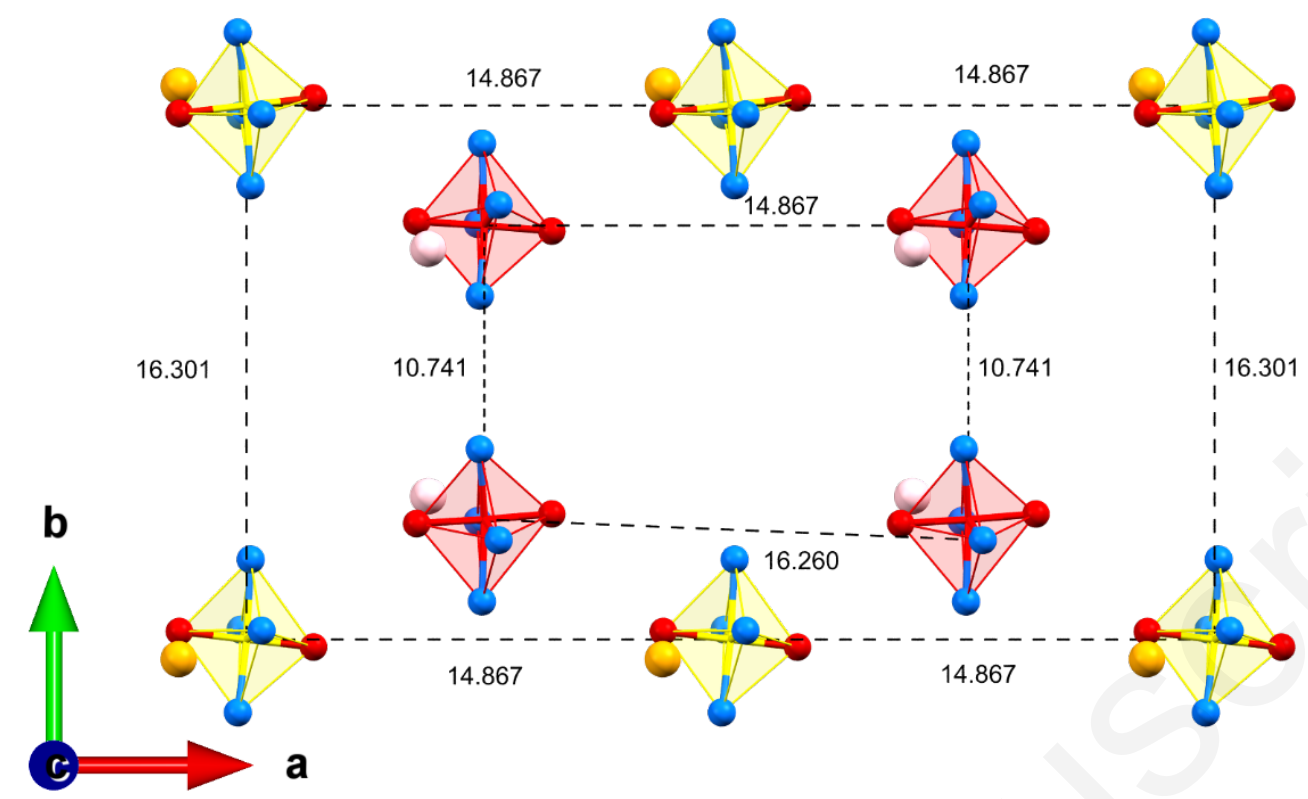

P1

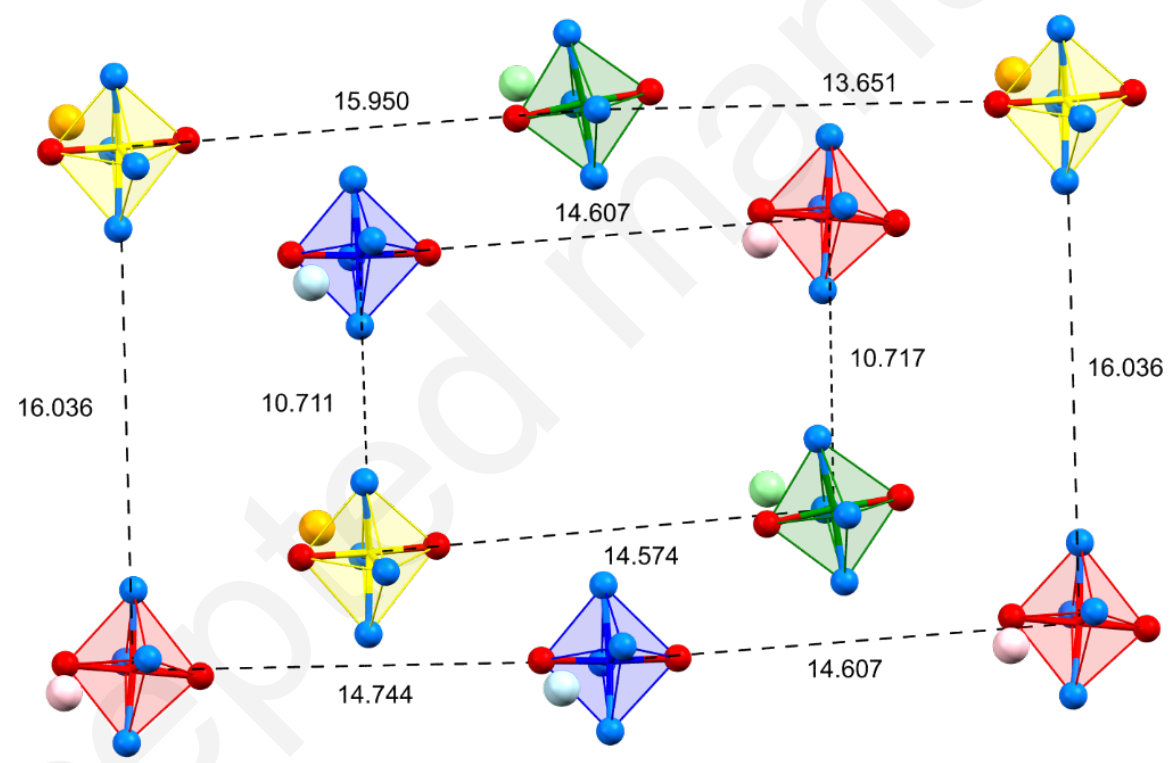

Figure S16. Same as figure S15 with different distances indicated. 


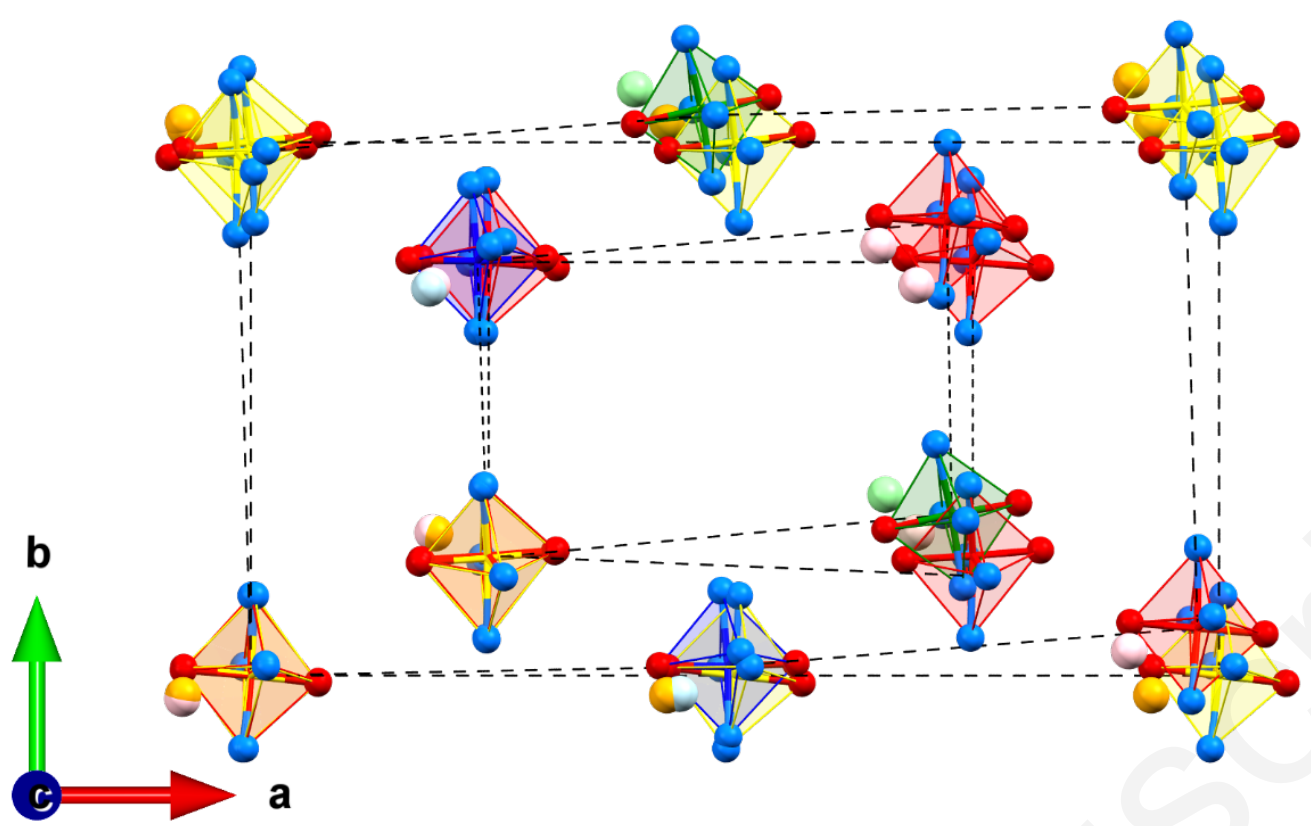

Figure S17. Overlay of molecules of the $P 1$ structure and $P c$ structure, showing local distortions of the $\mathrm{Mn}^{3+}$ cations (shown as polyhedra) and the boron atoms of the $\mathrm{BPh}_{4}^{-}$ counteranions (shown as balls). The overlay is viewed along the $c$ axis for the $P c$ structure. All other atoms are removed for clarity. 


\section{Density of states calculations}

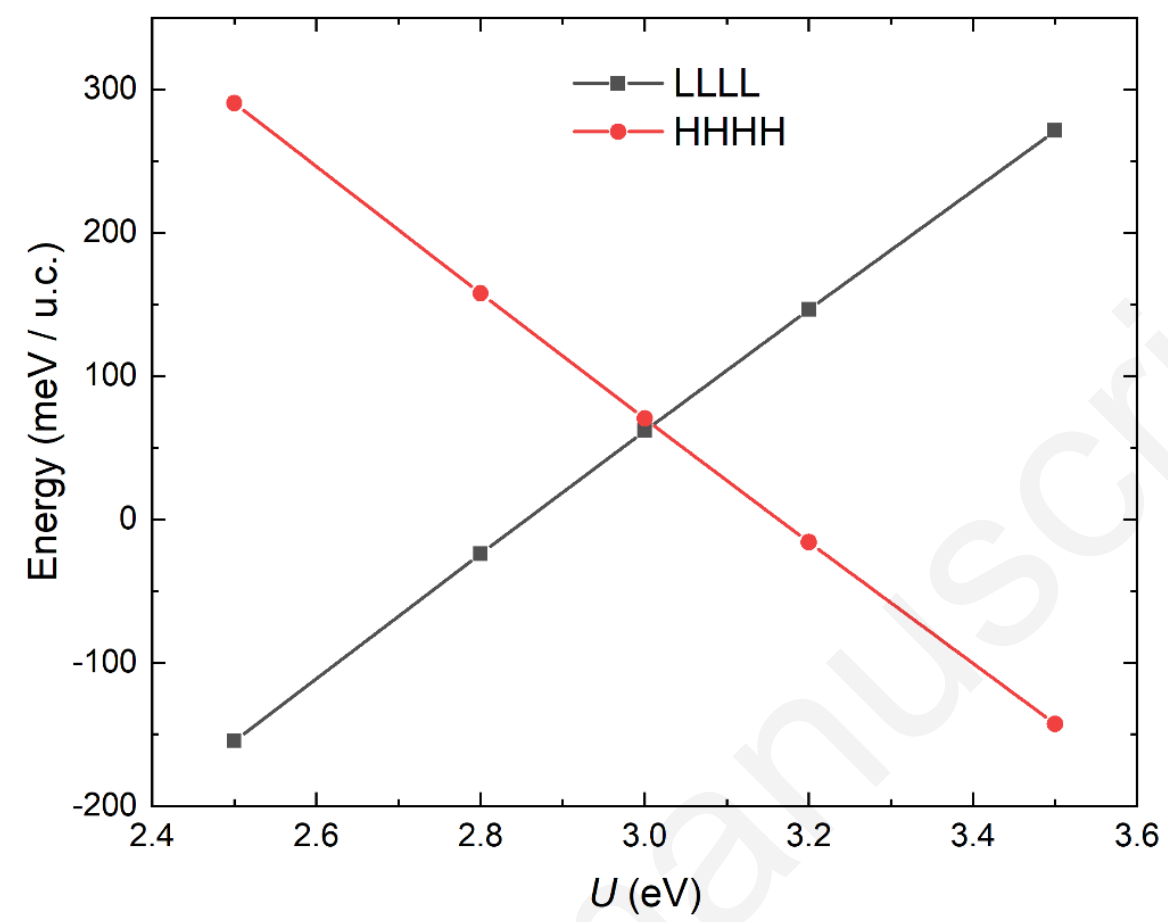

Figure S18. The $U$-dependent total energies of all LS state (LLLL) and all HS state (HHHH) related to the experimental ground state, the two LS and two HS state in HS-LS phase. $U$ around $3.0 \mathrm{eV}$ gives the result consistent with the experiments. 


\section{References}

1. Jakobsen, V. B.; Trzop, E.; Gavin, L. C.; Dobbelaar, E.; Chikara, S.; Ding, X.; Esien, K.; Müller-Bunz, H.; Felton, S.; Zapf, V. S.; Collet, E.; Carpenter, M. A.; Morgan, G. G., Stress-Induced Domain Wall Motion in a Ferroelastic Mn3+ Spin Crossover Complex. Angew. Chem. Int. Ed. 2020, 59 (32), 13305-13312.

2. Zapf, V. S.; Kenzelmann, M.; Wolff-Fabris, F.; Balakirev, F.; Chen, Y., Magnetically induced electric polarization in an organometallic magnet. Phys. Rev. B 2010, 82 (6), 060402.

3. Zapf, V. S.; Sengupta, P.; Batista, C. D.; Nasreen, F.; Wolff-Fabris, F.; Paduan-Filho, A., Magnetoelectric effects in an organometallic quantum magnet. Phys. Rev. B 2011, 83 (14), 140405.

4. Chikara, S.; Gu, J.; Zhang, X. G.; Cheng, H.-P.; Smythe, N.; Singleton, J.; Scott, B.; Krenkel, E.; Eckert, J.; Zapf, V. S., Magnetoelectric behavior via a spin state transition. Nat. Commun. 2019, 10 (1), 4043.

5. Detwiler, J. A.; Schmiedeshoff, G. M.; Harrison, N.; Lacerda, A. H.; Cooley, J. C.; Smith, J. L., Magnetization of UBe13 to 60 T. Phys. Rev. B 2000, 61 (1), 402-404.

6. Blöchl, P. E., Projector augmented-wave method. Phys. Rev. B 1994, 50 (24), 17953 17979.

7. Kresse, G.; Furthmüller, J., Efficiency of ab-initio total energy calculations for metals and semiconductors using a plane-wave basis set. Comput. Mater. 1996, 6 (1), 15-50.

8. Kresse, G.; Joubert, D., From ultrasoft pseudopotentials to the projector augmented-wave method. Phys. Rev. B 1999, 59 (3), 1758-1775.

9. Perdew, J. P.; Burke, K.; Ernzerhof, M., Generalized Gradient Approximation Made Simple. Phys. Rev. Lett. 1996, 77 (18), 3865-3868.

10. Grimme, S.; Antony, J.; Ehrlich, S.; Krieg, H., A consistent and accurate ab initio parametrization of density functional dispersion correction (DFT-D) for the 94 elements H-Pu. J. Chem. Phys 2010, 132 (15), 154104.

11. Resta, R., Macroscopic polarization in crystalline dielectrics: the geometric phase approach. Rev. Mod. Phys. 1994, 66 (3), 899-915.

12. Czyżyk, M. T.; Sawatzky, G. A., Local-density functional and on-site correlations: The electronic structure of La2CuO4 and LaCuO3. Phys. Rev. B 1994, 49 (20), 14211-14228. 